\title{
ESTRATIGRAFÍA DE POZOS COMO UN APORTE AL CONOCIMIENTO DE LA GEOLOGÍA DEL CUATERNARIO DEL VALLE DE EL GUARCO (CARTAGO), COSTA RICA
}

\author{
Enrique Quintanilla $^{1 *}$, Guillermo E. Alvarado ${ }^{1,2}$, Claudia Marín $^{1} \&$ Melissa Durán $^{1}$ \\ ${ }^{1}$ Escuela Centroamericana de Geología, Universidad de Costa Rica, \\ Apdo. 214-2050, Costa Rica. \\ ${ }^{2}$ Área de Amenazas y Auscultación Sísmica y Volcánica (A3SV), Instituto \\ Costarricense de Electricidad, Apdo. 10032-1000, San José, Costa Rica \\ * Autor para contacto: quikegeo@gmail.com
}

(Recibido: 03/03/08 ; aceptado: 03/05/08)

\begin{abstract}
The Guarco valley is known for being an intermountain depression which has been overfilled by volcanic products (i.e. debris flows, debris avalanches, lava flows) and volcano-sedimentary products (coluvial, fluvial and lake deposits) from Irazú volcano as well as from the adjacent Tertiary sedimentary and volcanic mountain ranges. A lithological correlation was established, based on the interpretation of 85 boreholes (all within depths of 31 to $200 \mathrm{~m}$ ). This correlation was established for three sectors, one to the west, other to the east and one at the central zone of the city of Cartago. The western sector showed a sedimentary basement of Miocene rocks, overlain by a series of lava flows $\sim 18 \mathrm{~m}$ thick. These lavas are overlain by at least 4 different events of alternated lahars and debris avalanches with a total thickness of $67 \mathrm{~m}$, separated by lenses of clays, which can be interpreted as paleosols. The top of the epiclastic sequence corresponds to a 6000 years old lacustrine and a debris avalanche deposit closing the Holocene. The central sector shows at the basement a series of lava flows $52 \mathrm{~m}$ thick, overlaid by $130 \mathrm{~m}$ thick laharic deposits. The eastern sector has up to $46 \mathrm{~m}$ thick lahar deposits overlain by $75 \mathrm{~m}$ of lava flows that are covered by lahars as well. At the top of the sequence, the distal facies of the Reventado debris avalanche are found, and they are restricted to the western sector and historic lahars mainly on rivers channels. The stratigrafic correlations show the existence of buried lava flows that are much more extensive than the morphology suggest, as well as the filling-up of the Middle to Upper Pleistocene basin with epiclastic products. Based on the stratigraphy and correlations with paleomastozoological founds, it can be interpreted that the Upper Pleistocene deposits (?) are at a very shallow depth in the southern part of the valley, while to the east, the Holocene deposits are dominant. Also, the stratigrafic profiles confirm the presence of the Aguacaliente Fault System that cuts through El Guarco Valley.

Keywords: Cartago, El Guarco Valley, Quaternary, volcano-estratigraphy, Aguacaliente fault.
\end{abstract}




\begin{abstract}
Resumen: El valle de El Guarco es una depresión intermontana, colmatada por productos volcánicos (avalanchas y flujos de detritos, coladas de lava) y volcano-sedimentarios (coluvios, depósitos lacustres y fluviales) procedentes del volcán Irazú y de los cerros sedimentarios adyacentes (calizas, lutitas, diques, coladas masivas y andesitas en almohadillas). Con base en la interpretación de 85 perforaciones (profundidades entre 31 y $200 \mathrm{~m}$ ), se ha realizado una correlación de litologías en tres sectores principales (occidente, centro y oriente) de la ciudad de Cartago y alrededores. El sector occidental mostró un basamento sedimentario del Mioceno seguido por una serie de coladas de lava de $18 \mathrm{~m}$ de espesor. Esta secuencia está sobreyacida por al menos 4 eventos, alternando lahares y debris avalanches (espesor total de $67 \mathrm{~m}$ ), separados por lentes arcillosos, que pueden interpretarse como paleosuelos. Un depósito lacustre - palustre y un depósito de debris avalanche de unos 6000 años de antigüedad (Unidad Reventado), coronan la secuencia en el Holoceno. El sector central muestra en su parte basal una serie de coladas de lava (52 m), seguida por depósitos laháricos (130 $\mathrm{m}$ de espesor total). El sector oriental se caracteriza igualmente por poseer lahares (46 m de espesor) sobreyacidos por $75 \mathrm{~m}$ de lava, a su vez cubierta por lahares. Al tope de la secuencia se encuentra la facies distal del debris avalanche del Reventado restringido al occidente, y lahares históricos mayoritariamente en los cauces. Las correlaciones estratigráficas evidencian la existencia de coladas de lavas sepultadas, más extensas de lo que la morfología sugiere, así como la paulatina colmatación de la cuenca en el Pleistoceno Medio-Superior por productos epiclásticos. Con base en la estratigrafía y correlación con hallazgos paleomastozoológicos, se puede interpretar que los depósitos del Pleistoceno Superior (?) están muy superficiales al sur del valle; mientras que hacia el oriente, predominan los depósitos del Holoceno. Además, los perfiles estratigráficos confirman la presencia del sistema de fallas de Aguacaliente, que cruza el valle de El Guarco.

Palabras clave: Cartago, Valle de El Guarco, Cuaternario, volcano-estratigrafía, falla Aguacaliente.
\end{abstract}

\section{INTRODUCCIÓN}

Cartago, capital de la provincia del mismo nombre, es una de las ciudades más pobladas de Costa Rica, con más de 132000 personas (Instituto Nacional de Estadísticas y Censos, INEC, ver www.inec.go.cr). Dicha ciudad se sitúa en el valle de El Guarco, (también denominado Valle de Coris y Cartago), una depresión morfológica intermontana colmatada por productos epiclásticos y efusivos. Limita al norte y oeste con el volcán activo Irazú, al oeste con la serranía de la Carpintera y al sur con las estribaciones de la cordillera de Talamanca. El valle ha sido aprovechado desde épocas precolombinas para el asentamiento y la agricultura. Actualmente ahí es donde se ubican importantes zonas industriales, comerciales y proyectos habitacionales. Esta región ha sido sometida a los embates de la naturaleza, particularmente a las crecidas del río Reventado, deslizamientos, terremotos y a la actividad del volcán Irazú.

El presente trabajo tiene como objetivo establecer la estratigrafía del valle de El Guarco con base en descripciones de perforaciones realizadas en esta zona por empresas privadas, para así obtener su disposición espacial y posibles espesores, además de su posible fuente u origen.
Adicionalmente, pretende mostrar una correlación con la estratigrafía propuesta en la literatura existente, así como sus correlaciones temporales basado en hallazgos paleontológicos y en las dataciones con radiocarbono realizadas en trabajos anteriores. Por otro lado, se realiza un aporte al estudio del fallamiento reciente de la zona basado en perfiles realizados.

\section{METODOLOGÍA}

Se recopilaron 85 reportes de perforación de pozos, ubicados en los alrededores de la ciudad de Cartago, que fueron aportados por el Servicio Nacional de Aguas Subterráneas, Riego y Avenamiento (SENARA). Con base en ellos, se elaboraron modelos en profundidad y correlaciones litológicas con el propósito de establecer la estratigrafía de la zona. En este punto es necesario hacer énfasis en las posibles variables de interpretación de cada uno de los profesionales a cargo de la descripción de cada pozo. Sin embargo, si es posible tomar los rasgos más importantes de cada una y llevar a cabo, de manera fiable, una correlación de este tipo.

El cuadro 1, muestra los códigos de los pozos utilizados para este trabajo y su distribución 
espacial se muestra en la figura 1. Para establecer la estratigrafía y la disposición de las unidades de la zona de estudio, se realizaron perfiles geológicos donde la densidad de pozos permitía una correlación adecuada y se dividió en zonas occidental, central y oriental. Algunas giras de reconocimiento fueron efectuadas con el fin de corroborar lo reportado en superficie por la literatura geológica.

\section{CONTEXTO GEOLÓGICO}

El volcán Irazú se localiza a $31 \mathrm{~km}$ al NE de Cartago, es el más alto del país con una elevación de 3432 m s.n.m. El Irazú es un escudo volcánico complejo, que posee una larga historia de erupciones prehistóricas e históricas. Su más reciente periodo eruptivo fue entre los años 1962-65 (Krushensky,

Cuadro 1

Perforaciones utilizadas en el presente trabajo.

\begin{tabular}{|c|c|c|c|c|c|c|c|c|c|c|c|}
\hline \multirow[t]{2}{*}{ Pozo } & \multicolumn{2}{|c|}{ Coordenadas } & \multirow[t]{2}{*}{$\operatorname{Prof}(m)$} & \multirow[t]{2}{*}{ Pozo } & \multicolumn{2}{|c|}{ Coordenadas } & \multirow[t]{2}{*}{$\operatorname{Prof}(m)$} & \multirow[t]{2}{*}{ Pozo } & \multicolumn{2}{|c|}{ Coordenadas } & \multirow[t]{2}{*}{$\operatorname{Prof}(m)$} \\
\hline & E & $\mathrm{N}$ & & & E & $\mathrm{N}$ & & & E & $\mathrm{N}$ & \\
\hline IS-289 & 541,8 & 204,5 & 180 & IS-43 & 535,5 & 205,2 & 48,77 & IS-222 & 542,2 & 203,0 & 87 \\
\hline IS-17 & 541,5 & 205,2 & 49,5 & IS-18 & 577,5 & 219,2 & 48,76 & IS-224 & 541,9 & 203,3 & 95 \\
\hline IS-11 & 541,5 & 209,2 & 30,5 & IS-22 & 544,5 & 205,1 & 42,67 & IS-232 & 540,0 & 203,0 & 70 \\
\hline IS-110 & 546,8 & 203,2 & 100 & IS-299 & 543,5 & 204,7 & 24 & IS-295 & 542,1 & 202,7 & 31 \\
\hline IS-174 & 544,1 & 203,7 & 60 & IS-134 & 543,3 & 202,9 & 132 & IS-237 & 537,9 & 205,1 & 150 \\
\hline IS-233 & 539,6 & 203,0 & 80 & IS-14 & 543,4 & 206,3 & 32 & IS-207 & 540,3 & 203,0 & 74 \\
\hline IS-283 & 538,2 & 205,5 & 128 & IS-31 & 541,9 & 204,9 & 44,2 & IS-217 & 540,6 & 209,8 & 80 \\
\hline IS-109 & 546,5 & 205,6 & 200,1 & IS-85 & 541,8 & 205,3 & 31 & IS-117 & 541,5 & 203,0 & 80 \\
\hline IS-119 & 544,2 & 205,1 & 42 & IS-150 & 545,5 & 203,6 & 150 & IS-110 & 546,3 & 203,0 & 90 \\
\hline IS-314 & 538,0 & 205,4 & 150 & IS-208 & 546,7 & 202,7 & 74 & IS-39 & 520,5 & 202,2 & 30,5 \\
\hline IS-230 & 542,8 & 207,3 & 70 & IS-16 & 580,5 & 240,2 & 51 & IS-218 & 542,1 & 201,8 & 30,5 \\
\hline IS-294 & 539,8 & 204,2 & 46 & IS-251 & 541,3 & 204,7 & 89 & IS-298 & 539,7 & 202,5 & 36 \\
\hline IS-257 & 542,0 & 203,3 & 43 & IS-255 & 541,7 & 204,1 & 85,87 & IS-176 & 542,8 & 204,5 & 56 \\
\hline IS-12 & 542,5 & 209,2 & 61 & IS-256 & 542,3 & 203,2 & 34 & IS-87 & 543,5 & 202,9 & 50 \\
\hline IS-252 & 541,3 & 204,8 & 86 & IS-266 & 540,0 & 203,4 & 80 & IS-44 & 535,5 & 227,5 & 48 \\
\hline IS-26 & 544,7 & 205,8 & 49 & IS-272 & 539,4 & 203,4 & 75 & IS-189 & 540,1 & 203,7 & 82 \\
\hline IS-115 & 542,6 & 209,8 & 58 & IS-290 & 541,0 & 204,5 & 90 & IS-188 & 540,8 & 204,9 & 35 \\
\hline IS-168 & 545,6 & 203,5 & 90 & IS-20 & 545,6 & 202,2 & 48,77 & IS-167 & 539,0 & 202,6 & 90 \\
\hline IS-19 & 526,5 & 217,2 & 54 & IS-223 & 542,2 & 203,3 & 82 & IS-136 & 542,0 & 207,1 & 90 \\
\hline IS-304 & 548,2 & 206,6 & 34 & IS-235 & 547,1 & 205,0 & 46 & IS-317 & 549,7 & 208,3 & 100 \\
\hline IS-297 & 548,5 & 202,6 & 136 & IS-234 & 539,3 & 203,2 & 84 & IS-308 & 548,1 & 206,9 & 60 \\
\hline IS-205 & 544,0 & 203,2 & 59 & IS-105 & 545,2 & 203,2 & 131,8 & IS-61 & 548,8 & 203,2 & 60,9 \\
\hline IS-165 & 549,7 & 203,2 & 162 & IS-69 & 548,5 & 203,5 & 127,2 & IS-48 & 224,2 & 570,5 & 37,2 \\
\hline IS-164 & 549,7 & 203,4 & 177 & IS-67 & 548,9 & 203,3 & 90 & IS-47 & 560,5 & 255,2 & 53 \\
\hline IS-162 & 549,5 & 202,9 & 90 & IS-66 & 547,1 & 201,9 & 18,9 & IS-45 & 530,5 & 285,2 & 48,8 \\
\hline IS-122 & 545,3 & 202,2 & 34 & IS-445 & 205,2 & 545,7 & 74 & IS-495 & 204,7 & 543,8 & 30 \\
\hline IS-513 & 204,9 & 545,3 & 80 & IS-292 & 204,7 & 543,3 & 136,5 & IS-27 & 205,2 & 545,0 & 30,5 \\
\hline IS-324 & 204,8 & 539,9 & 152 & IS-343 & 204,8 & 541,2 & 184 & IS-545 & 204,6 & 541,2 & 22,5 \\
\hline
\end{tabular}




\section{Mapa de ubicación y geológico del valle del Guarco, Cartago}
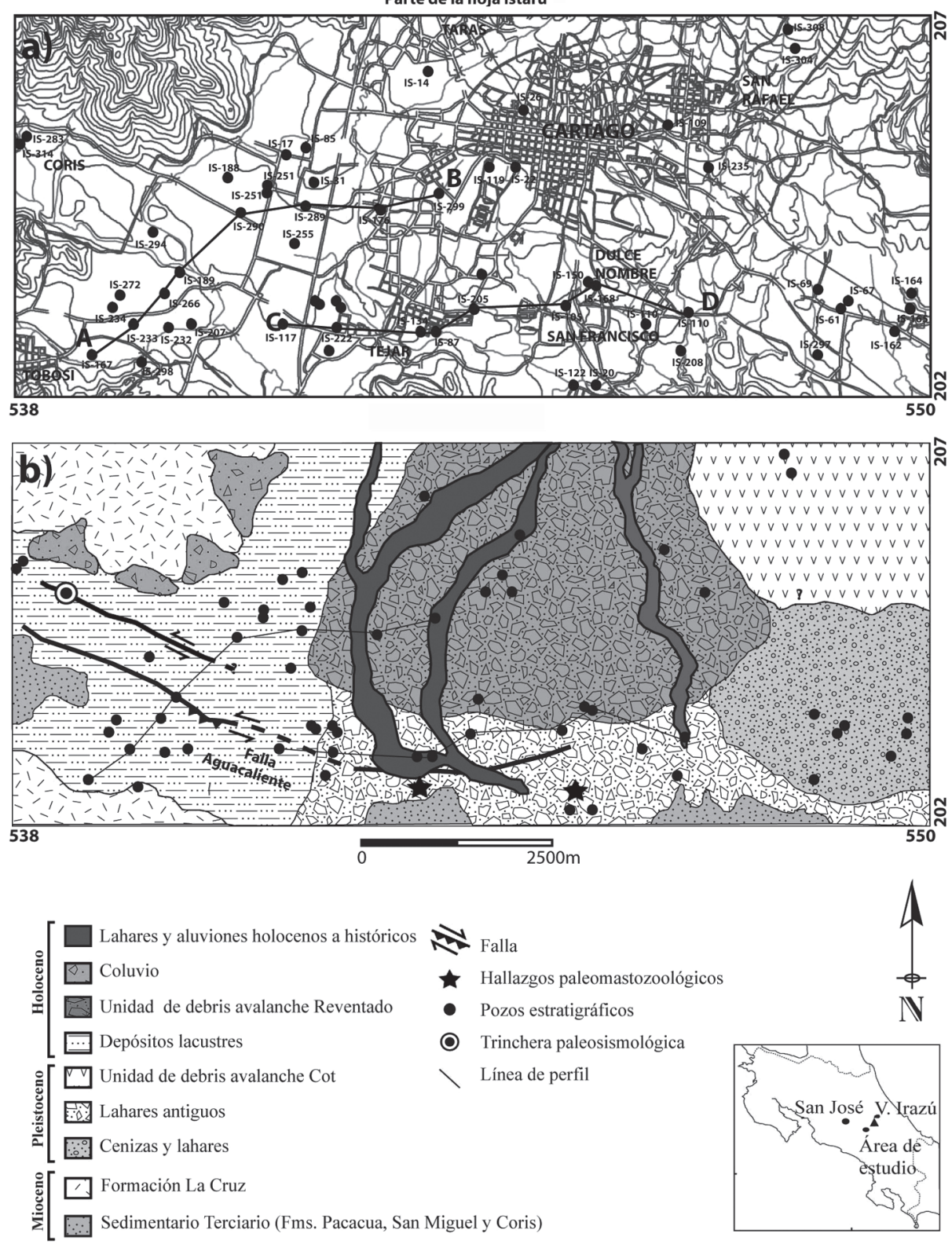

Fig. 1: A) Ubicación de pozos utilizados y orientación de perfiles. B) Mapa geológico del valle de El Guarco, Cartago. Las curvas topográficas provienen de parte de la hoja Istarú 1:50 000, IGN. Modificado de Krushensky (1972), Alvarado et al. (2006), Pavanelli et al. (2004). El trazado de la falla Aguacaliente se modificó con base en Montero \& Kruse (2006).

1972). Quizá uno de los primeros trabajos geológicos de la zona fue de Segura \& León (1941), quienes hacen referencia a rasgos geomorfológicos y a las lagunas remanentes. Los depósitos laháricos y lacustres de Cartago y alrededores fueron estudiados por Dóndoli \& Torres (1954), quienes lo denominaron lavina. Algunos depósitos inconsolidados que se extienden hacia la propia ciudad de Cartago, 
han sido interpretados y descritos como producto de un debris avalanche datados con radiocarbono en $\sim 5930 \pm 100$ años (Pavanelli et al., 2004), cuya edad calibrada (Reimer et al., 2004) sería de 6493-7005 años a.P., así como lahares recientes hasta históricos (Alvarado \& Schmincke, 1994). Ignimbritas, lavas y epiclastos del Pleistoceno han sido descritos por Krushensky (1972), Alvarado (1986, 1994), y Alvarado et al. (2006). Dóndoli \& Torres (1954) y Krushensky (1972) describen una llanura fluviolacustrina al oeste del río Reventado y al sur de los cerros de la Carpintera, que consisten en arenas cuarzosas, limos y arcillas finamente estratificadas, producto de la erosión de los cerros sedimentarios terciarios. Segura \& León (1941) afirman que uno de los últimos vestigios de dicho lago son las lagunas pantanosas de Coris y el Cortijo.

Badilla et al. (1999) describen coluvios en las márgenes de los ríos Navarro y Aguacaliente, en referencia a un depósito de clastos angulares a subangulares, polilitológico, mal seleccionado, en una matriz areno-arcillosa anaranjada, café y rojiza, con restos de materia orgánica. Los lahares que se distribuyen en los alrededores de El Tejar y San Francisco, presentan bloques métricos de lavas provenientes del Irazú. Los aluviones están constituidos por bloques de areniscas cuarzosas, volcarenitas, lavas e intrusivos provenientes de los cerros adyacentes.

$\mathrm{Al}$ sur del valle de El Guarco, muy cerca del límite del área de estudio, en los alrededores de Aguacaliente de Cartago, se hallaron restos de megafauna pleistocena extinta. El primer hallazgo se reportó en 1921 durante la construcción de un acueducto para la planta eléctrica de Cartago, paralelo al río Aguacaliente, en donde se encontraron restos óseos, algunos de ellos correspondieron con un mastodonte (Tristán, 1920). En 1960 se encontraron a unos $65 \mathrm{~m}$ al este del puente de Aguacaliente, otros restos de mastodonte (Echeverría, 1960). Laurito (1988) analizó un fragmento del ramo mandibular derecho del mastodonte reportado por Tristán y correspondió a un Cuvieronius hyodon, macho y adulto. Los restos del mastodonte reportado en 1921 se encontraron a 1,5 m de profundidad, en un suelo aluvial (Tristán, 1920). Alvarado (1986) describe el depósito en dicha localidad como un lahar del Cuaternario Superior. Más recientemente, en 1999, fueron hallados los restos de un caballo fósil, clasificado por Valerio \& Laurito (2004) como un Equus cf. E. conversidens. Dichos autores lo asocian por correlación paleontológica con otros lugares de Norteamérica como del Pleistoceno Superior Tardío. Sin embargo, Kurtén \& Anderson (1980) extienden el rango temporal de esta especie desde el Irvingtoniano al Rancholabreano; es decir abarca todo el Pleistoceno. En la descripción elaborada por Valerio \& Laurito (2004) desgraciadamente no se incluye el tipo de sedimentos o suelo en que fueron encontrados.

En tiempos históricos recientes, el valle de El Guarco además de haber sido afectado por las avenidas laháricas del volcán Irazú, también ha sido afectado por fuertes terremotos que han destruido en repetidas ocasiones la ciudad de Cartago. Muchos de estos eventos sísmicos han sido generados por la falla activa de Aguacaliente-Orosi, como es el ejemplo del terremoto de 1910 (Montero \& Miyamura, 1981; Montero et al., 2005).

\section{DESCRIPCIONES VOLCANO- ESTRATIGRÁFICAS}

La secuencia estratigráfica presente en el valle del Guarco y descrita en este trabajo fue establecida mayoritariamente con base en las descripciones de las litologías encontradas en pozos y en algunas observaciones de campo. Con base en las unidades estratigráficas presentes, la cuenca fue dividida en tres zonas: occidental, central y oriental.

\section{Zona Occidental}

La base de la secuencia al sur consiste en rocas sedimentarias que se encuentra a una profundidad de $66 \mathrm{~m}$, con un espesor mínimo de $7 \mathrm{~m}$ (pozo IS-167). Hacia el norte las rocas más basales encontradas son lavas, con un espesor mínimo de $18 \mathrm{~m}$ (pozo IS-289) y que se encuentran en las perforaciones a partir de los $60 \mathrm{~m}$ b.n.s. (Fig. 2). Ambas litologías se encuentran sobreyacidas en la zona central por depósitos laháricos antiguos que presentan mayores espesores hacia el NE (67 $\mathrm{m}$, pozo IS-290), y son separados por horizontes arcillosos relativamente continuos. 
La parte alta de la secuencia fue interpretada con base en la geología de superficie y de pozos, como constituida por depósitos de debris avalanche de menos de $24 \mathrm{~m}$ de espesor (IS-299) hacia el norte, y depósitos lacustres hacia el sur con un espesor máximo de $68 \mathrm{~m}$ (IS-233).

El presente trabajo fue enfocado a los depósitos cuaternarios, por lo que las formaciones rocosas cercanas no fueron estudiadas en detalle. Sin embargo, se realizó un reconocimiento de campo para verificar aspectos morfológicos y las formaciones superficiales. Se identificó la existencia de lavas en almohadillas, en las cercanías del valle de Coris (539412E/205116N), correlacionables con la Formación La Cruz de Denyer \& Arias (1991). Se presentan como cuerpos decimétricos de andesitas, con forma ovoide irregular, fuertemente meteorizadas y tectonizadas, con contactos relativamente nítidos, separados por un material silíceo y óxidos de hierro de aspecto vidrioso (brillo graso o jasperoide), que podría corresponder con hialoclastita. Algunas de las almohadillas presentan morfologías similares a pedúnculos en un extremo y una forma cóncava hacia arriba hacia el otro extremo (Fig. 4A y 4B). Su presencia es novedosa, aunque no sorprendente, puesto que: a) la Fm. La Cruz es concordante con las rocas sedimentarias miocenas infrayacentes (ver Denyer \& Arias, 1991), b) las rocas volcánicas de Talamanca son en su mayoría coetáneas con las rocas sedimentarias miocenas (McMillan et al., 2004), y c) se encontraron rocas sedimentarias miocenas intercaladas en la secuencia volcánica del Cerro de la Muerte (Ian McMillan, com. escrita, 2003), así como peperitas (halladas por G.E. Alvarado), respectivamente.

\section{Correlación propuesta}

Los pozos IS-167 y IS-298, al NE de la localidad de Tobosi (Fig. 1a) reportaron la presencia de rocas estratificadas a una profundidad de $66 \mathrm{y}$ 36 m b.n.s., respectivamente. Las características físicas de la roca y su disposición espacial, sugieren que estos depósitos sedimentarios corresponden con la Formación Coris que aflora al sur del valle del Guarco.

Las lavas en el NE del perfil, podrían asociarse con el volcán Irazú, debido a su cercanía, posición cronoestratigráfica y litología. Además pueden asociarse con la Unidad Reventado, definida por Alvarado et al. (2006), reportada con una edad de $136 \pm 5 \mathrm{ka}$ basada en dataciones con ${ }^{40} \mathrm{Ar} /{ }^{39} \mathrm{Ar}$.

Los depósitos laháricos que sobreyacen las lavas pueden asociarse con varios eventos antiguos separados entre sí por lentes arcillosos correspondientes con paleosuelos, que conforman el abanico de Cartago. El depósito superior ubicado al norte, puede asociarse por su morfología con los depósitos distales del debris avalanche de la Unidad Reventado, de edad Holocena, descrito, mapeado y datado en su parte media por Pavanelli et al. (2004).

Los depósitos arcillosos podrían corresponder con paleosuelos de depósitos lacustres que, según Dóndoli \& Torres (1954), se formaron debido al represamiento del río Reventado. Justamente en dichos suelos, datados en $3665 \pm 145$ años a.P. (Montero, 1991; Woodward \& Clyde, (1993), se ha encontrado cerámica precolombina del período Pavas (300 a.C.-300 d.C.), cuya edad calibrada (Reimer et al., 2004) sería de 3640-4415 años a.P. Los perfiles realizados para este artículo, sugieren

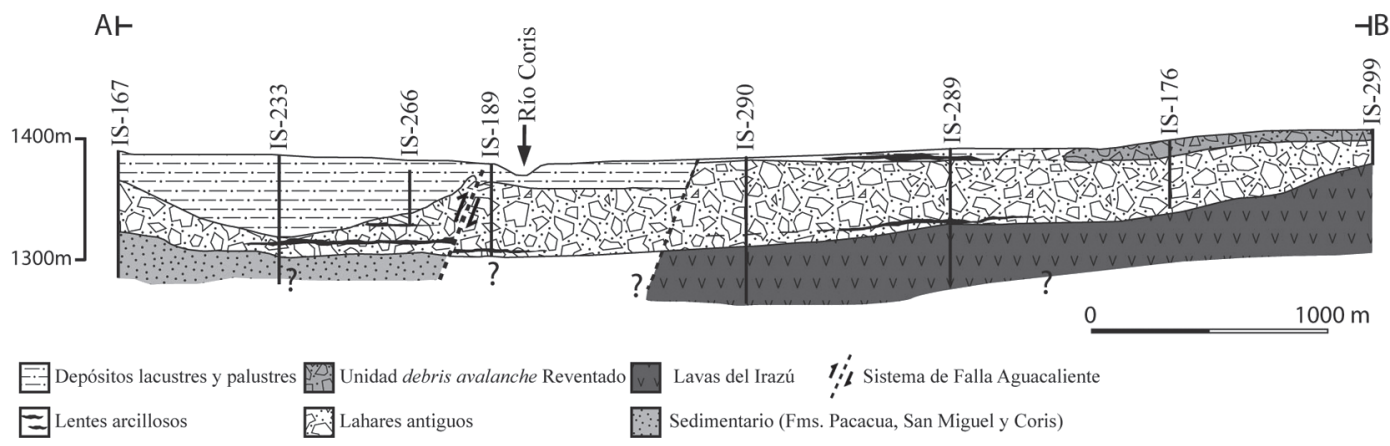

Fig. 2: Perfil estratigráfico idealizado del sector occidental. La línea punteada muestra la posible disposición de la traza de falla Aguacaliente en este sector. 


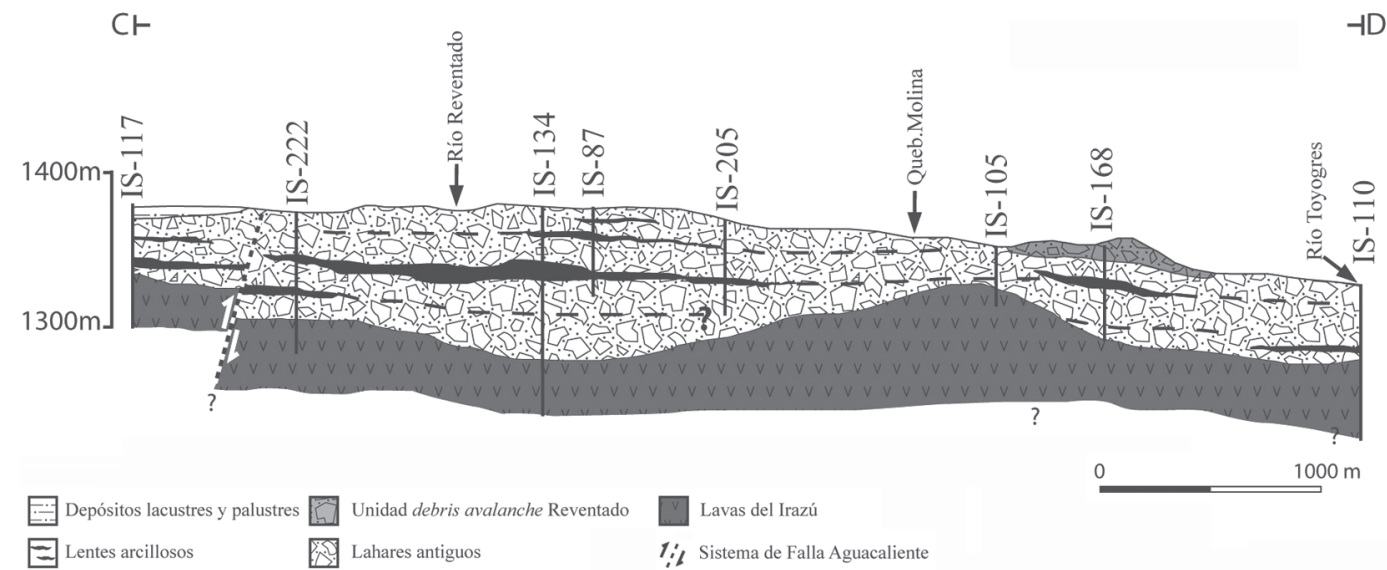

Fig. 3: Perfil estratigráfico idealizado del sector central. El perfil muestra la traza de la falla Aguacaliente, y su desplazamiento se ve reflejado en la colada de lava basal y al menos en dos paleosuelos.

que la datación del depósito lacustre corresponde con la parte más superficial de dicho depósito, pues por el espesor que alcanzan, es de esperar que se hayan depositado desde el Pleistoceno Superior? y hasta el Holoceno.

\section{Zona Central}

Este sector se encuentra representado en la base por unas secuencia lávica de un espesor mínimo de 52 m (IS-110), sobreyacida por depósitos laháricos de hasta $130 \mathrm{~m}$ de espesor (IS-134) con intercalaciones de horizontes arcillosos. La parte superior consiste en depósitos de debris avalanche, se encuentran en la parte superior de la secuencia, además de una delgada capa de no más de $10 \mathrm{~m}$ de depósitos lacustres, restringidos a la parte oriental del sector central.

\section{Correlación propuesta}

La estratigrafía y disposición de las lavas sugieren que estas provienen del volcán Irazú. Las secuencias laháricas, corresponden con depósitos antiguos que, al igual que en el sector occidental, se encuentran intercalados con horizontes arcillosos asociados a paleosuelos, definiendo al menos 4 eventos diferentes (ver Fig. 4c). El depósito de debris avalanche se interpreta como el depósito distal de la unidad Reventado.
Estas correlaciones concuerdan con la paleotopografía (Fig 3), ya que se puede observar que las depresiones que se formaron entre los eventos lávicos del volcán Irazú (Pleistoceno Medio- Superior?), fueron rellenadas por depósitos laháricos, dejando una topografía más plana. Además, los depósitos distales del debris del Reventado muestran una estructura de hummocky con colinas de al menos $10 \mathrm{~m}$ de alto, típicas de estos depósitos.

\section{Zona Oriental}

La densidad de pozos disponibles en esta zona no es la mejor, sin embargo, se logró establecer una estratigrafía simplificada, pero no así perfiles geológicos confiables como en las otras zonas.

La base de esta zona consiste en una secuencia de lahares e intercalaciones de capas arcillosas, de espesor mínimo de $46 \mathrm{~m}$ (pozo IS-165) y que aparece a partir de los $116 \mathrm{~m}$ b.n.s. La secuencia está sobreyacida por una lava, con un espesor máximo de $75 \mathrm{~m}$, que puede encontrarse a $15 \mathrm{~m}$ de profundidad en el pozo IS-162. Sobre la secuencia de lavas se encuentran una serie de depósitos laháricos antiguos (con espesores máximos de 64 m en el pozo IS-109), muestran variaciones laterales de espesor en toda la zona, y aparecen a partir de $1 \mathrm{~m}$ de profundidad en la mayoría de los pozos. 

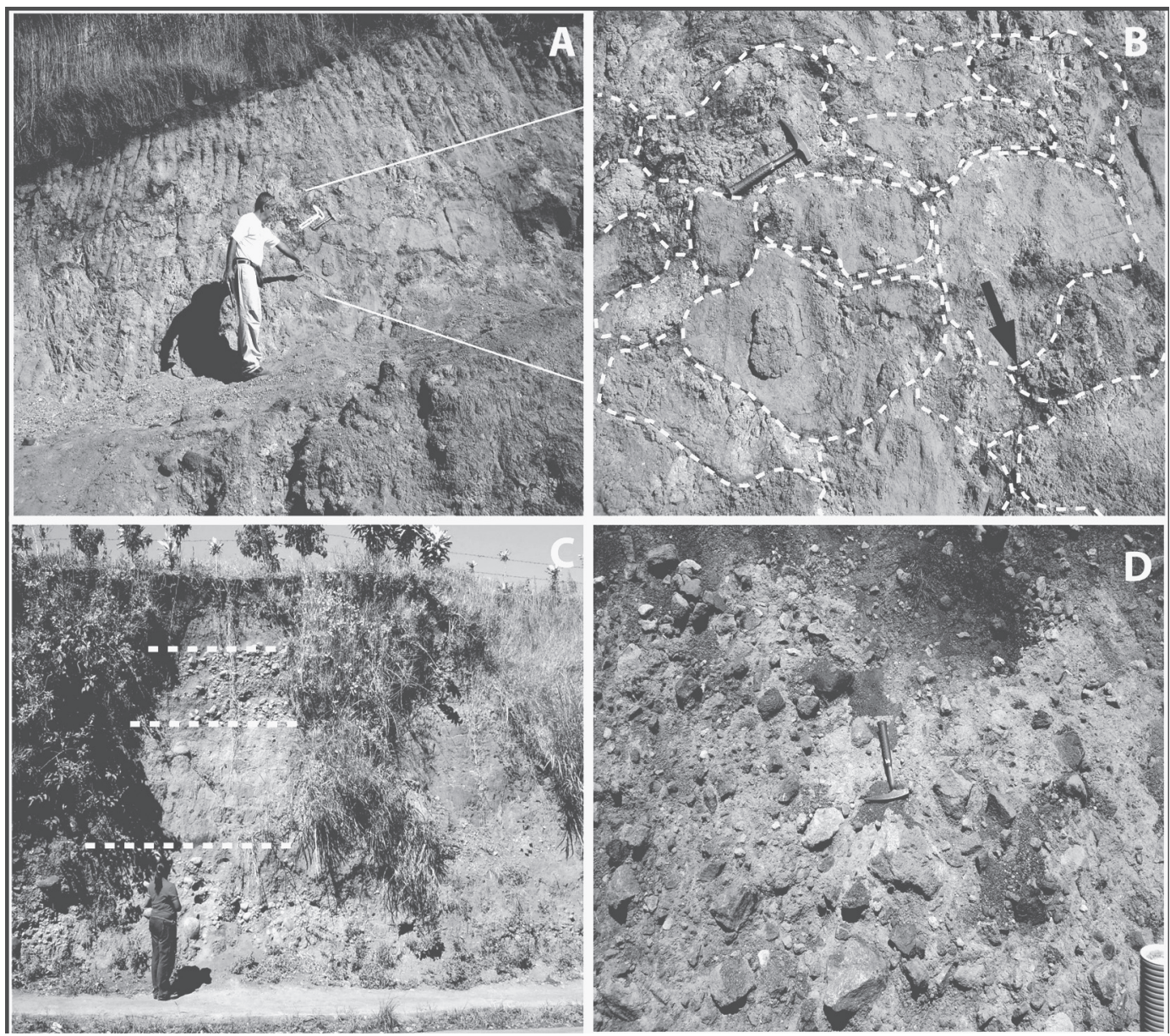

Fig. 4: A. Afloramiento de las posibles lavas almohadilladas en el valle de Coris (539412E/205116N). B. Detalle de las almohadillas. La línea punteada blanca muestra su disposición, la flecha muestra uno de los pedúnculos más claros. C. Depósitos epiclásticos holocenos aflorando en las cercanías de Dulce Nombre en la margen derecha del río Toyogres. Se distinguen al menos cuatro niveles de lahares. D. Depósito de bloques decimétricos a métricos, angulosos, tanto frescos como hidrotermalizados correspondientes a la Unidad Debris Avalanche Reventado.

En el campo, estas litologías presentan morfologías relativamente planas, con el desarrollo de un suelo amarillento. En algunos afloramientos, se puede observar en suelos mediana a profundamente desarrollados la presencia de estructuras remanentes de lapilli acrecional.

\section{Correlación propuesta}

Las lavas pueden estar asociadas a la actividad del volcán Irazú debido a su conexión morfológica con un frente de lava observable al NE de
Cartago (Fig 1), específicamente con la Unidad Paraíso o con la Unidad Reventado-Sapper, descritas por Alvarado et al. (2006).

Los lahares superiores podrían tener como origen la actividad más reciente del volcán Irazú y se pueden correlacionar con los lahares de la Unidad Cot descritas por Pavanelli et al. (2004). Estos lahares son previos a la colada de Cervantes, lo que los ubicaría en el Pleistoceno Superior (ver Alvarado et al., 2006). Varios niveles de depositación están separados por lentes arcillosos. En algunas zonas, especialmente hacia el oeste de este sector (pozos IS-109 y IS-235), los lahares 
podrían ser equivalentes a los depósitos distales del debris del Reventado, tal y como lo sugiere la morfología y en correspondencia con lo mapeado por Pavanelli et al. (2004).

Al límite sur del área de estudio, se hallaron restos fósiles de mastodontes y de caballos (Tristán, 1920; Alvarado, 1986, 1994; Valerio \& Laurito, 2004), a menos de $2 \mathrm{~m}$ de profundidad. Esto sugiere que el Pleistoceno Superior está muy superficial en dicho sector y el Holoceno está restringido al suelo reciente. La ubicación de los hallazgos paleontológicos se muestra en la Fig. 1b.

\section{CONSIDERACIONES NEOTECTÓNICAS}

Desde hace varias décadas diversos autores han propuesto la existencia de un sistema de fallas que cortan el Valle del Guarco, que tiene como estructura principal el sistema de fallas de Aguacaliente (también llamadas de Coris y Guarco). Este sistema de fallas se considera sísmicamente activo y fue el responsable de la destrucción de Cartago en 1910 (Montero \& Miyamura, 1981), entre otros. En 1991 se realizó una trinchera de $112 \mathrm{~m}$ de longitud en el Valle de Coris (Fig 1b) con el fin de estudiar la falla Aguacaliente. En ella se observaron una serie de fallas normales e inversas de alto ángulo, que fueron interpretadas como fallas asociadas con la estructura principal de desplazamiento sinestral. Este movimiento afecta varios niveles lacustres y de suelos, uno de ellos datado en $3665 \pm 145$ años (Montero, 1991; Woodward \& Clyde, 1993). Estudios geomorfológicos y geofísicos recientes, consideran que el movimiento es predominantemente sinestral con componente normal (Montero \& Kruse, 2006).

Los niveles estratigráficos de las perforaciones estudiadas, son muy recientes (Pleistoceno Medio?, mínimo Pleistoceno Superior a Holoceno) y, por ello, el neotectonismo no es tan evidente puesto que no ha desplazado mucho las capas, más aún si las fallas tienen una componente predominante de desplazamiento de rumbo.

Pese a que la densidad de perforaciones no es la deseada, las mismas permiten dilucidar varios aspectos importantes. Por ejemplo, el perfil de la zona occidental mostró una estructura de grada en los niveles arcillosos que puede ser interpretada como una falla con un movimiento secundario aparente inverso en la zona en la que la traza de la falla Aguacaliente (Figs. 1 y 2). Además, corresponde con el límite oriente de la cuenca lacustrina. Esto refuerza la propuesta de Fernández \& Montero (2002), quienes sugieren que dicha falla tiene componentes inversos en ciertos sectores especialmente hacia al sur de la ciudad de Cartago, aspecto que fue también verificado en la trinchera realizada en 1991. Tampoco contradice la propuesta de Montero \& Kruse (2006), ya que los autores también señalan la presencia de sectores de transpresión local asociados a un posible sistema "pull- apart" en los valles de Coris y El Guarco. Taylor (1994) señala la existencia también de sistemas de fallas de rumbos sinuosos al norte del valle de Coris, que podrían estar asociados al sistema Aguacaliente, y presentan estructuras de sillas de fallas, espolones truncados y fuentes termales alineadas.

El perfil realizado para la zona central del área de estudio, el cual corta transversalmente el abanico aluvial de Cartago (Fig. 1a), mostró la presencia de niveles de lavas y paleosuelos desplazados en correspondencia con la traza de la falla Aguacaliente. En ambos casos el movimiento es aparentemente inverso con un desplazamiento de al menos $20 \mathrm{~m}$, dependiendo de la inclinación real de la falla en profundidad.

\section{CONCLUSIONES}

Tres sectores con estratigrafías similares y diferencias menores fueron definidos en El valle del Guarco. La zona occidental tiene un basamento de rocas sedimentarias (Fig. 5) correlacionadas con las formaciones Pacacua, San Miguel y Coris del Mioceno y rocas volcánicas extrusivas correlacionadas con la Formación La Cruz. Estas rocas volcánicas incluyen posibles lavas en almohadilla que sugieren la existencia de un vulcanismo submarino coetáneo con las rocas sedimentarias Miocenas, muchas de ellas enriquecidas en componente volcánico. Esto confirmaría la cercanía de focos eruptivos activos con la cuenca sedimentaria, lo cual había sido postulado por varios autores desde hace varias décadas. 
Estos eventos miocénicos, fueron seguidos por coladas de lava del Pleistoceno Medio pero particularmente del Pleistoceno Tardío. Depósitos lacustres especialmente al occidente de la depresión, en las cercanías del valle de Coris, se depositaron a partir del posible represamiento del río Reventado. La parte superior de la secuencia la conforman depósitos holocénicos de debris avalanche correspondientes con la Unidad Reventado y por coluvios locales.

El sector central muestra una estratigrafía similar, donde la base de la secuencia se encuentra representada por flujos de lava de al menos $52 \mathrm{~m}$ de espesor, sobreyacidos por al menos 4 depósitos laháricos de $130 \mathrm{~m}$ de espesor, separados por paleosuelos. La parte superior mostró estructuras de hummocky las cuales son indicadores geomorfológicos de depósitos de debris avalanche de Reventado.

El sector oriental está compuesto por depósitos epiclásticos en la base, con espesores importantes de hasta $46 \mathrm{~m}$ asociados a la actividad Pleistocena Media (?) del volcán Irazú, seguida por lavas de al menos $75 \mathrm{~m}$ de espesor. En el tope de la secuencia se encuentran espesores (usualmente 30-46 m de espesor) de lahares que aparecen a pocos $\mathrm{m}$ de profundidad. Depósitos distales del debris avalanche de Reventado se restringen hacia el occidente.

De las perforaciones se desprende que los depósitos más jóvenes (Holocenos) están en el sector nórdico, occidental y central del Valle del Guarco, mientras que al oriente y al sur, la edad de los sedimentos incrementa, hasta probablemente el Pleistoceno Superior. En estos últimos se encontraron fósiles de mastodontes y caballos extintos a menos de $2 \mathrm{~m}$ de profundidad.

La interpretación geológica de los pozos y la correlación mediante perfiles en la zona occidental y central, sugieren un posible desplazamiento de la falla Aguacaliente.

\section{Columna estratigráfica compuesta para el valle de El Guarco}
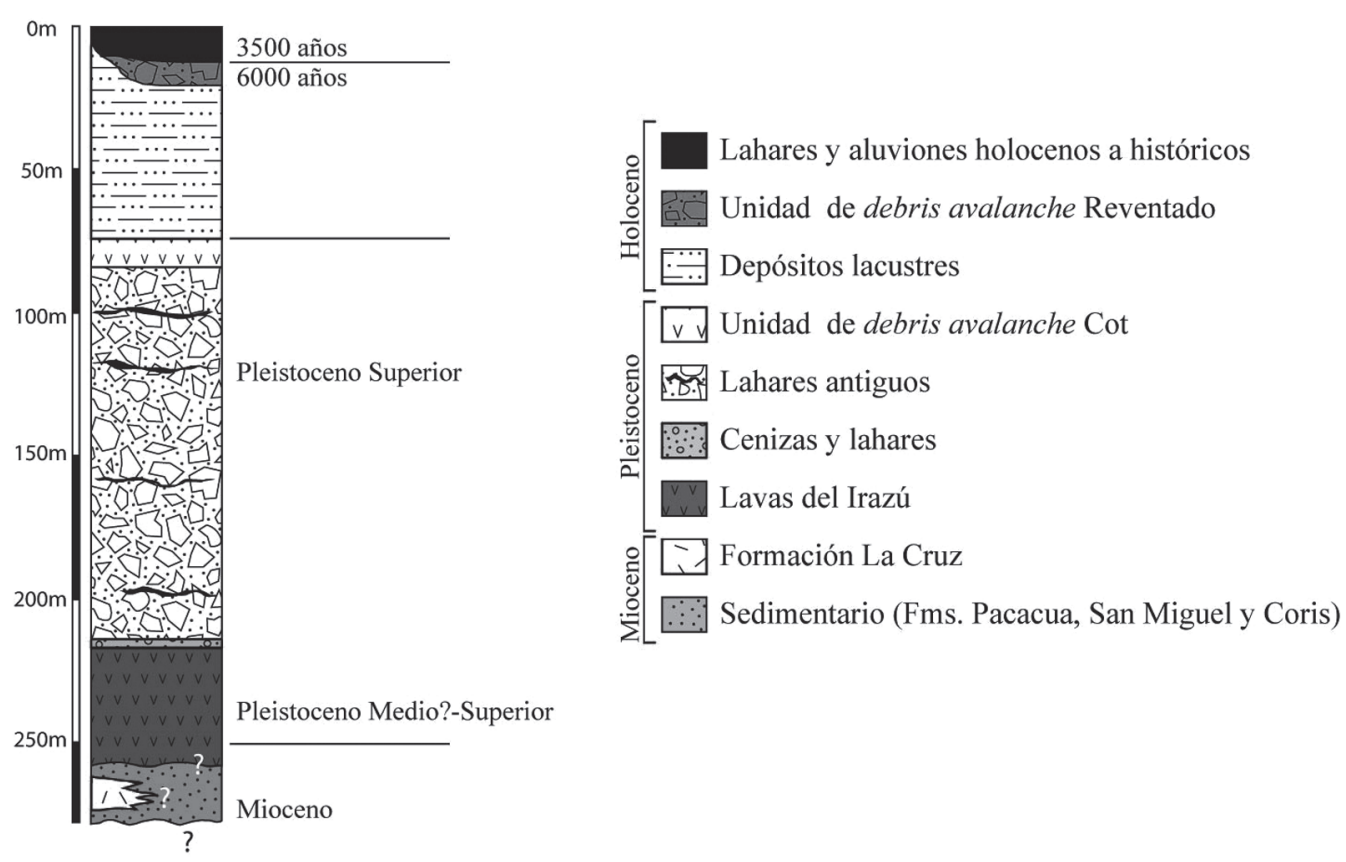

Fig. 5: Columna estratigráfica compuesta para el valle del Guarco. Muestra la disposición cronoestratigráfica de las litologías identificadas en los pozos analizados. 


\section{AGRADECIMIENTOS}

El presente trabajo fue posible gracias a la colaboración ofrecida por SENARA. Se agradece la ayuda brindada para la elaboración de los modelos en profundidad a Carlos Ramírez. Los sismólogos Walter Montero y Waldo Taylor aportaron información valiosa. El colega Oscar Lücke amablemente colaboró con la traducción del resumen y Gerardo Soto aportó valiosas sugerencias al primer borrador. Los revisores Sigfried Kussmaul y Paulo Hidalgo, así como un revisor anónimo, mejoraron sustancialmente el presente trabajo. Este trabajo se inició como una investigación del curso de Vulcanología (G-5120) en el primer semestre de 2006.

\section{REFERENCIAS}

ALVARADO, G.E., 1986: Hallazgos de megamamíferos fósiles en Costa Rica.- Rev. Geol. Amér. Central, 4: 1-46.

ALVARADO, G.E., 1993: Volcanology and petrology of Irazú Volcano, Costa Rica. 261 págs. Univ. Kiel [Tesis de Doctorado].

ALVARADO, G.E., 2000: Los volcanes de Costa Rica: Geología, historia y riqueza natural. 284 págs, EUNED (2 edición). San José, Costa Rica.

ALVARADO, G.E. \& SCHMINCKE, H., 1994: Stratigraphy and sedimentology of the raintriggered lahars from the 1963-1965 Irazú Eruption, Costa Rica. -Zbl. Geol. Paläont. Teil 1 (H.1/2): 513-539

ALVARADO, G.E., CARR, M. J., TURRIN, B., SWISHER, C., SCHMINCKE, H. \& HUDNUT, K., 2006: Recent volcanic history of Irazú volcano, Costa Rica: Alternation and mixing of two magma batches, and pervasive mixing. Geological Society of America: 262-263.
BADILLA, E., LINKIMER, L., ZUÑIGA, H., 1999: Geología de Navarro y alrededores, Provincia de Cartago, Costa Rica. 100 págs., Escuela de Geología, Univ. Costa Rica [Tesis de Bachillerato].

DENYER, P. \& ARIAS, O., 1991. Estratigrafía de la región central de Costa Rica. Rev. Geol. América Central, 12:1-59

DÓNDOLI, C. \& TORRES, A. 1954: Estudio geoagronomómico de la región oriental de la Meseta Central. -180 págs, Ministerio de agricultura e Industria, San José.

ECHEVERRÍA, J., 1960: Aguacaliente cementerio de mastodontes. La Nación, 18-IX1960, p. 12; San José Costa Rica.

FERNÁNDEZ, M. \& MONTERO, W., 2002: Fallamiento y sismicidad del área entre Cartago y San José, Valle Central de Costa Rica. - Rev. Geol. Amér. Central, 26:25-37.

HIDALGO,P.,ALVARADO, G.E. \& LINKIMER, L., 2004: La Lavina del Valle Central (Costa Rica): ¿lahar o debris avalanche?. -Rev. Geol. Amér. Central, 30: 101-109.

KURTÉN,B.\&ANDERSON,E.,1981:Pleistocene mammals of North America. - xvii +443 págs., Columbia Univ. Press, New York.

KRUSHENSKY, R., 1972: Geology of the Istarú Quadrangle, Costa Rica. -U.S. Geol. Survey, Bull. 1358: iv + 46 págs., Washington, DC.

LAURITO, C., 1988: Los proboscídeos fósiles de Costa Rica y su contexto en la América Central. -Vínculos, 14 (1-2): 29-58.

McMILLAN, I.; GANS, P. \& ALVARADO, G., 2004: Middle Miocene to present plate tectonic history of the southern Central American Volcanic Arc. -Tectonophysics, 392: 325-348. 
MONTERO, W., 1991: Informe del componente fallas activas, Proyecto de Mitigación de riesgos sísmicos y volcánicos (MIRVYS). - vii + 39 págs, apéndices y mapas. Informe final. Informe interno.

MONTERO, W., BARAHONA, M., ROJAS, W. \& TAYLOR, M., 2005: Los sistemas de falla Agua Caliente y Río Azul y relevos compresivos asociados, Valle Central de Costa Rica. - Rev. Geol. Amér. Central, 33: 7-27.

MONTERO, W. \& MIYAMURA, S., 1981: Distribución de intensidades y estimación de los parámetros focales de los terremotos de Cartago de 1910, Costa Rica, América Central.- Informe Semestral, Inst. Geogr.. Nac., 2: 9-34.

MONTERO, W \& KRUSE, S., 2006: Estudio geotectónico y geofísico de la falla Aguacaliente en los valles de Coris y el Guarco. -Rev. Geol. Amér. Central, 34-35: 43-58.

PAVANELLI,N.,CAPACCIONI,B.,SAROCCHI, D., CALDERONI, G., VASELLI, O., TASSI, F., DUARTE, E., 2004: Geology and stability of the southern flenk of Irazú volcano, Costa Rica. -Acta Vulcanológica, sección especial, 16(1): 2004.

REIMER, P.J., HUGHEN, K.A., GUILDERSON, T.P., MCCORMAC, F.G., BAILLIE, MGL., BARD, E., BARRATT, P.,
BECK, J.W.,BROWN, D.M., BUCK, C.E., DAMON, P.E., RIEDRICH, M., KROMER, B., RAMSEY, C.B., REIMER, R.W., REMMELE, S., SOUTHON, J.R., STUIVER, M., VAN DER PLICHT, J., 2002: Preliminary Report of the first workshop of the IntCal04 Radiocarbon Calibration/Comparison Working Group. Radiocarbon 44, 653-61.

SEGURA, A. \& LEÓN, A., 1941. El Valle de Cartago y Coris. -Departamento Nacinal de Agricultura (DNA), 438-449.

TAYLOR, W., 1994: Características sismo-dinámicas del suelo blando en la región central de la ciudad de Cartago, Costa Rica. - 69 págs., Univ. Costa Rica [Tesis de Licenciatura].

TRISTÁN, J., 1920: Notas sobre los restos de un vertebrado fósil en Aguacaliente de Cartago. -Rev. Costa Rica, 2:337-340.

VALERIO A. \& LAURITO, C., 2004: Paleofauna de Aguacaliente de Cartago, Costa Rica. Parte I: Equus cf. E. conversidens Owen, 1869. -Rev. Geol. Amér. Central, 31: 87-92.

WOODWARD-CLYDE, 1993. A preliminary evaluation of earthquake and volcanic hazard significent to the major population centres of the Valley Central, Costa Rica. - 71 págs. + apéndices +mapas, Informe interno. 


\title{
HISTORICAL TEPHRA-STRATIGRAPHY OF THE COSIGÜINA VOLCANO (WESTERN NICARAGUA)
}

\author{
Petr Hradecký* \& Vladislav Rapprich \\ Czech Geological Survey (CGS), Klárov 3, 11821 Prague, Czech Republic \\ * Autor para contacto: petr.hradecky@geology.cz
}

(Recibido 11/04/07; aceptado 03/05/08)

\begin{abstract}
New detailed geological field studies and ${ }^{14} \mathrm{C}$ dating of the Cosigüina Volcano (westernmost Nicaragua) allow to reconstruct a geological map of the volcano and to establish a recent stratigraphy, including three historical eruptions. Five major sequences are represented. I: pyroclastic flows around $1500 \mathrm{AD}$, II: pyroclastic flows, scoria and pumice flows and surges, III: pyroclastic deposits related to a litoral crater, IV: pyroclastic flows related to 1709 AD eruption, and finally, V: pyroclastic deposits corresponding to the cataclysmic $1835 \mathrm{AD}$ phreatic, phreatomagmatic and subplinian eruption, which seems to be relatively small-scale in comparison with the preceding historical eruptions. The pulsating geochemical character of the pyroclastic rocks in the last five centuries has been documented. The beginning of every eruption is marked by increasing contents of silica and Zr. Based on that, regardless of present-day volcanic repose, the entire Cosigüina Peninsula should be considered as a very hazardous volcanic area.
\end{abstract}

Key words: Cosigüina volcano, tephra-stratigraphy, Nicaragua, Fonseca Gulf, ${ }^{14} \mathrm{C}$ dating.

RESUMEN: Nuevos estudios de campo detallados realizados en la zona del volcán Cosigüina (occidente de Nicaragua), junto con dataciones ${ }^{14} \mathrm{C}$, permitieron la reconstrucción de un mapa geológico y de la estratigrafía reciente de los depósitos piroclásticos, incluyendo tres erupciones históricas. Se encontraron cinco secuencias mayores. I: flujos piroclásticos cerca del año 1500 dC; II: flujos piroclásticos, flujos de escoria y pómez y oledas piroclásticas; III: depósitos piroclásticos relacionados con un cráter litoral; IV: flujos piroclásticos relacionados con la erupción del año 1709 dC, y finalmente, V: los depósitos piroclásticos correspondientes a la erupción cataclísmica del año 1835 dC, de carácter freático, freatomagmático y subpliniano, que parece ser de pequeña escala en comparación con las previas erupciones. Los productos analizados de las erupciones de los últimos cinco siglos revelan el carácter pulsante del contenido de $\mathrm{SiO} 2$ y Zr. A pesar de que el volcán se encuentra en estado de calma por más de 170 años, por su registro de erupciones violentas, así como por dicha variación química del magma, la península de Cosigüina puede considerarse como un área de alto peligro volcánico.

Palabras claves: Volcán Cosigüina, tefraestratigrafía, Nicaragua, Golfo de Fonseca, datación ${ }^{14} \mathrm{C}$.

HRADECKÝ, P. \& RAPPRICH, V., 2008: Historical tephra-stratigraphy of the Cosigüina volcano (Western Nicaragua). - Rev. Geol. Amér. Central, 38: 65-79. 


\section{INTRODUCTION}

Cosigüina Volcano is a broad volcanic shield lying on the westernmost limits of the Nicaraguan territory (e.g. Weyl, 1980), being part of the volcanic belt genetically associated with subduction of the Cocos plate beneath the Caribbean plate (Mann, 1995 and references therein). It is best known by its 1835 eruption (Mc Birney \& Williams, 1965; Williams, 1952; Self et al., 1989). The 700-m-deep crater has been formed by several violent explosive events. The presentday volcano rests on remnants of an older basaltic-andesite stratovolcano (Pre-Cosigüina stage), presumably of Pleistocene age, similarly to the eroded San Juan volcano located nearby.

Cosigüina Volcano is located at the Nicaraguan side of the Fonseca Gulf. The origin of this gulf, separating the Nicaraguan and Salvadorian segments of the Central American volcanic belt, is by the intersection of three first-order tectonic structures (Fig. 1). Such complicated tectonic setting is the result of the oblique subduction on the Central American trench. Steep angle of subduction is responsible for the extension and formation of the Central Graben (Salvadorian C.G. and Nicaraguan Depression). The oblique convergence of lithospheric plate leads to strike-slip of the Chortis block to the east along the Polochic-Motagua-Jocotán fault system (Burkart \& Self, 1985). Heterogeneous rates of this eastward motion rebound in the formation of the north-south trending grabens and the northeast-southwest trending Guayape left-lateral strike-slip fault (Finch \& Ritchie, 1991). In the area of Fonseca Gulf, the Guayape fault breaks the Central Graben and displaces the Salvadorian part to the southwest from the Nicaraguan segment. The Guayape fault is probably also responsible for the northwest limit of the Cosiguiina peninsula.

\section{Previous research}

Recent publications focus predominantly on the 1835 eruption of Cosiguiina (Fig. 2) and its impact on society and atmosphere. Various authors have documented the products of the
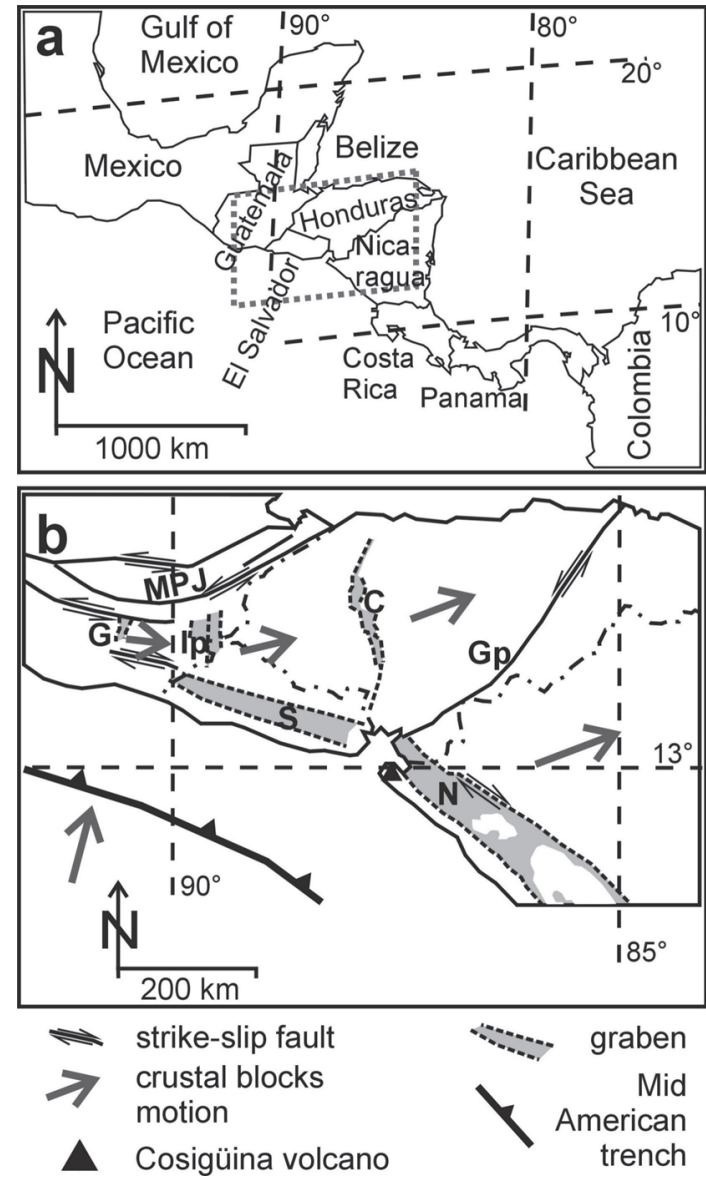

Fig. 1: Location of the study area and relations to the principal tectonic structures: C - Comayagua Graben, $\mathrm{G}$ - Guatemala City Graben, Gp - Guayape fault system, Ip - Ipala Graben, MPJ-Motagua-Polochic-Jocotán fault system, N-Nicaraguan Depression, S - Salvadorian Central Graben (simplified after Burkart \& Self, 1985).

youngest eruptions from the southern and eastern slopes of the edifice (e.g. Self et al., 1989; Scott et al., 2006). The previous evolution of this volcano was in contrast studied scarcely and if so, only briefly. Williams (1952) reported more than 30 lava flows exposed in the vertical crater walls with separating tuffs layers. Close to the hacienda Los Pozos on the southern outskirts of the volcano, he described basaltic-andesite lavas with plg $>$ cpx $>$ op $x>$ ol $>$ mt, with some olivine xenoliths (currently not confirmed). Similar rocks are 

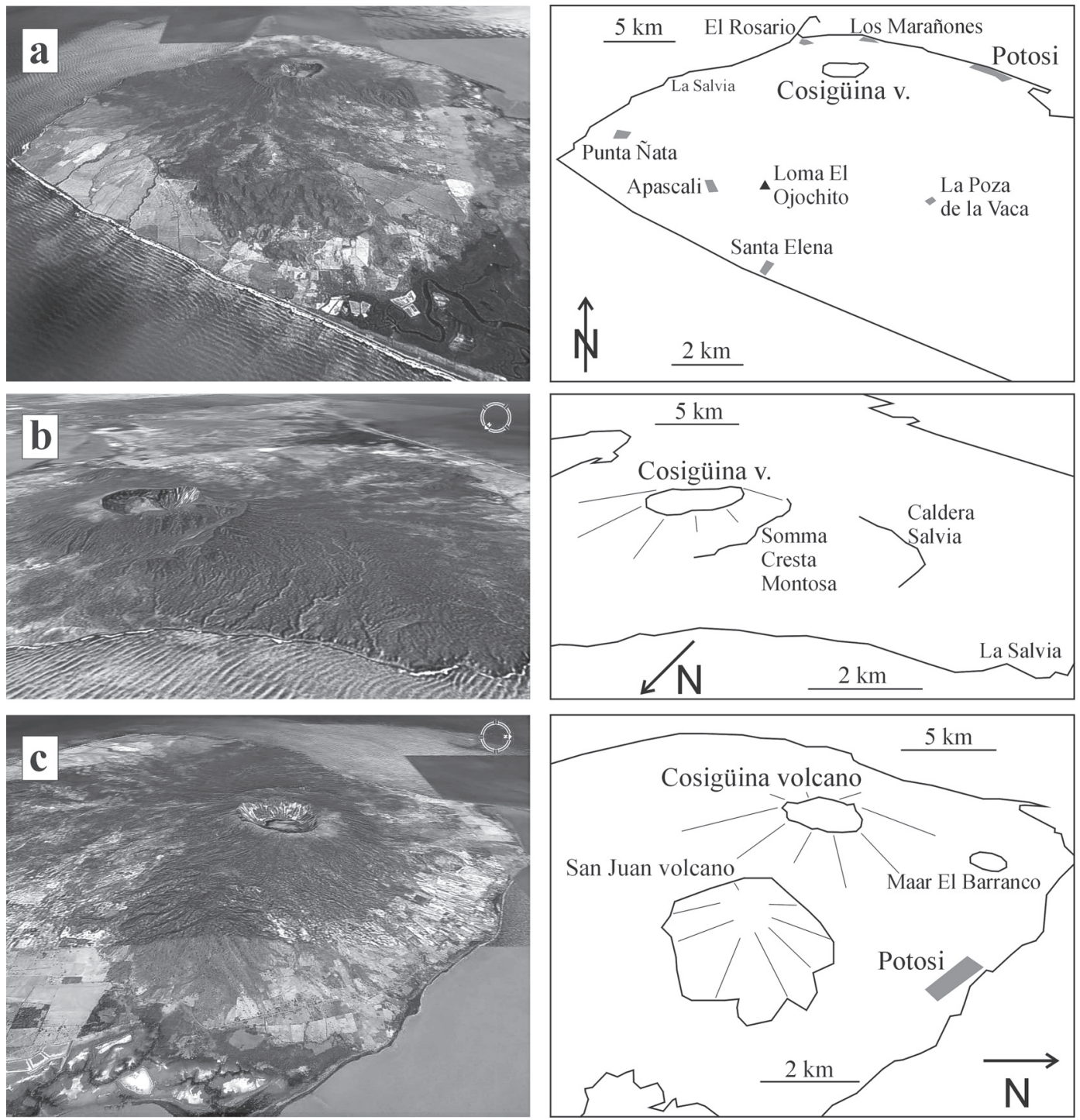

Fig. 2: Satellite images of the Cosigüina Volcano (source: www.earth.google.com) seen from a) south; b) northwest; c) east. Locations mentioned in text are marked. Images are in perspective, therefore scale-bars for front and rear are given.

shown in the photogeological map of "Catastro e Inventario de Recursos Naturales" (1972). Martínez \& Viramonte (1982) made a revision of the existing data on the volcano, but did not present any new data. Hradecký (1988) described the cliff section Punta Nata (now inaccessible) where the lowermost layers contained numerous buried animal bones, unfortunately not studied by any biologist or palaeontologist, and therefore not assigned to animal species.

General data were published in several summarizing works, e.g. by Williams (1972) and Weyl (1980), but lacking any detail description.

Self et al. (1989) summarized all available data from sections of the youngest deposits, stated that the 1835 famous eruption had 
commenced with phreatic activity, continued as a plinian eruption and finished as phreatic again. The analyzed rocks correspond to andesites. Scott et al. (2006) comprehensively studied the youngest - 1835 A.D. - eruption with paying only minor attention on previous activities. This author-team supposed, those eruption closely preceding eruption were of smaller magnitude then the famous 1835 A.D. one. They also reported some deposits of highmagnitude volcanic eruption, which is supposed to be at least few thousands years old.

Modern geological mapping (Fig. 3) accompanied with a new volcano-stratigraphic scheme (Fig. 4) have been compiled based on three weeks of field research held by the Czech geological expedition in 2001 (Hradecký et al., 2001).

\section{Methodology}

This paper summarizes the results of field and analytical research carried out by the team of Czech geologists - mainly from the Czech
Geological Survey. Over 60 outcrops were documented comprising 18 key profiles documented in detail (namely localities along "farallones" or cliffs on the northwest limits of the Cosiguiina peninsula). Individual defined volcaniclastic layers were correlated on the basis of volcanologic, lithologic and "overall-shape" characteristics (Fig. 5). The 1835 deposits were unfruitful for correlations, as this eruption buried all previous sequences over an erosional unconformity and therefore offer the only information that all other deposits are older. On the other hand, deposits of "dramatic surges" - DS were easily recognizable and their position in the middle part of the entire succession was really helpful especially on the western side of the volcano. For the elaboration of the map, the products of 1835 eruption were omitted from locations, where these are thinner than 0.5 $\mathrm{m}$. Otherwise, the map would appear unisonous and consisting solely of the 1835 tuffs, without such remedy. Nevertheless the 1835 tephra is ever present in low thickness all around the peninsula.

Twenty representative rock samples, of which sixteen belong to the proper Cosigüina Volcano, were

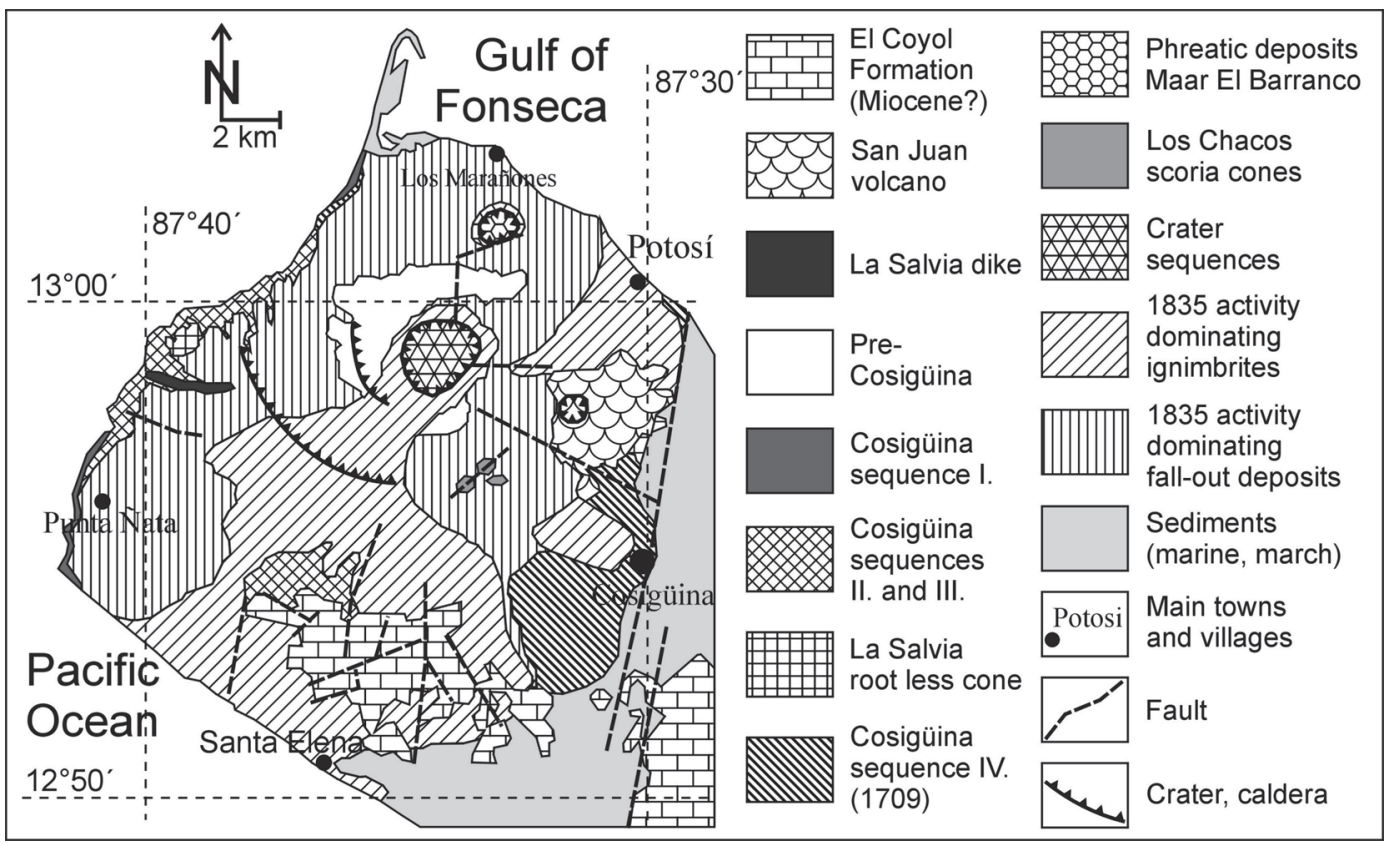

Fig. 3: Generalized geological map of the Cosigüina Volcano (simplified after Hradecký et al., 2001). 


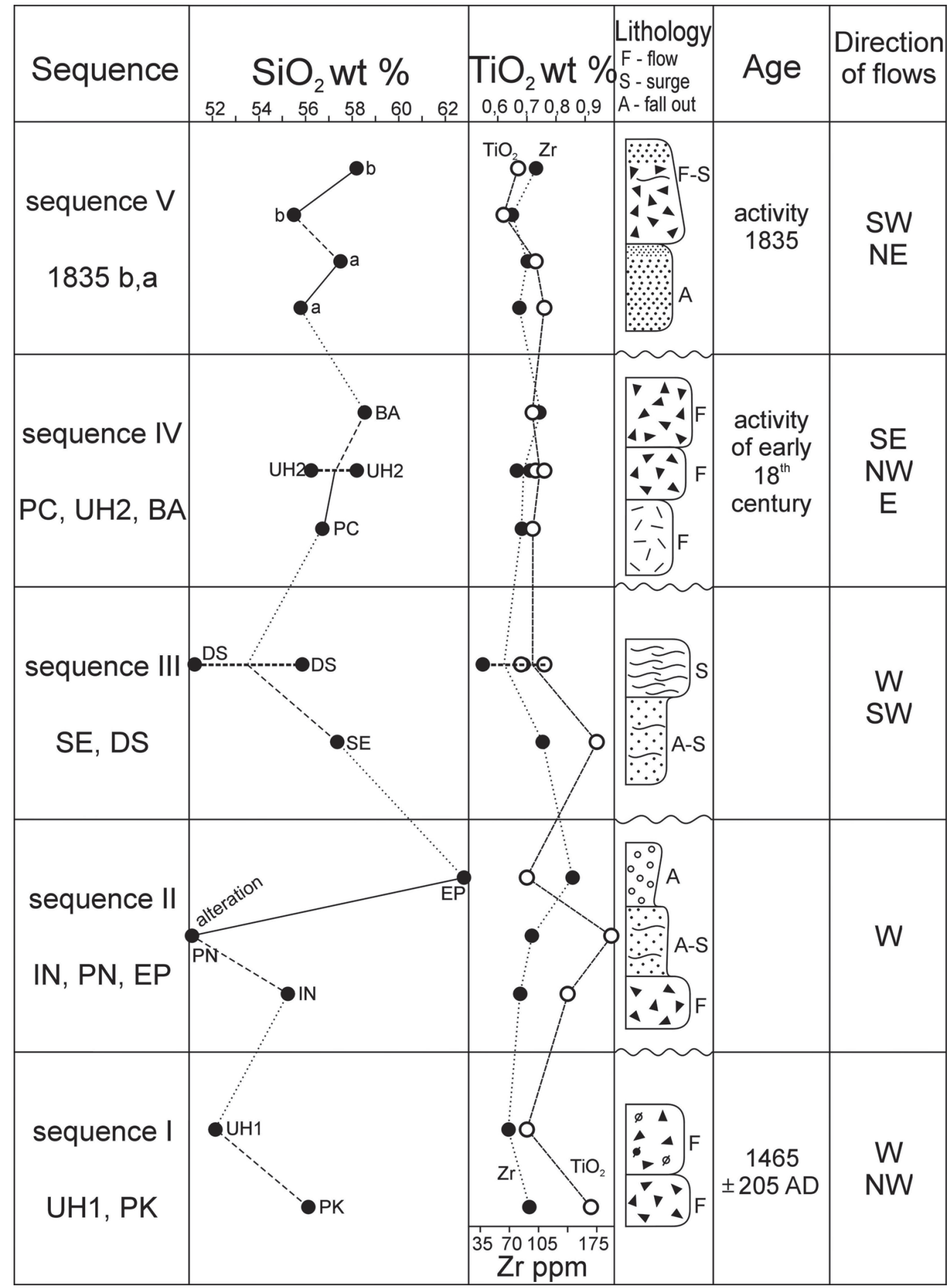

Fig. 4: Stratigraphic scheme of the explosive history of the Cosigüina Volcano. Stratigraphic and radiometric data accompany the main geochemical characteristics of the pyroclastic sequences. 


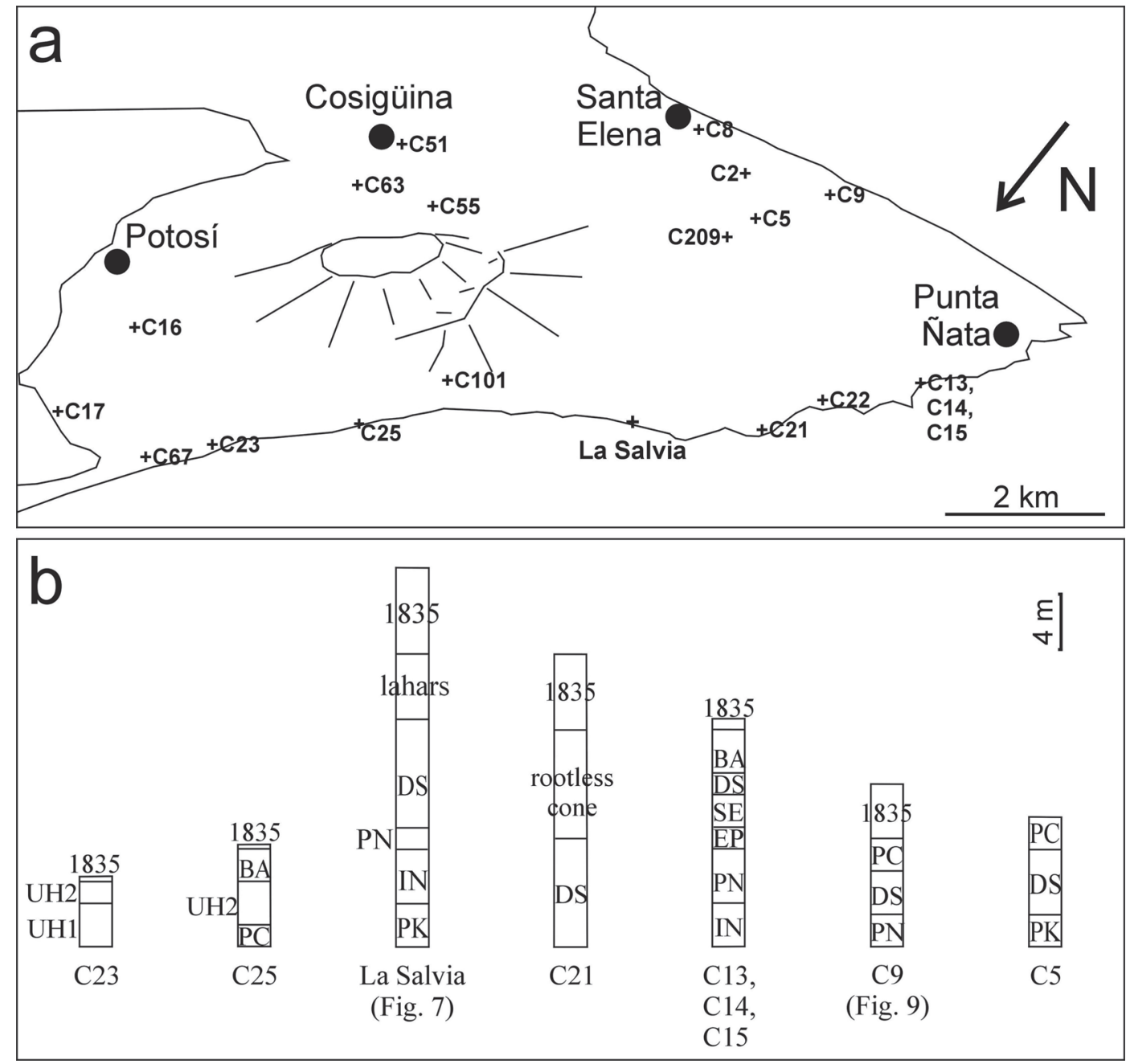

Fig. 5: a) Location of sampling- and detailed documentation sites (view from the NW). b) Correlation of main pyroclastic layers around the Cosigüina Peninsula.

analyzed in labs of the Czech Geological Survey in Prague. Silicate analyses were supplemented with selected trace elements determined using FAAS and XRF techniques. Analytical data were recalculated to water-free phase and plotted into diagrams in the GCDkit software (Janoušek et al., 2006). The common classification diagram of Le Bas et al. (1986), recommended by IUGS, was combined with the diagram of Winchester \& Floyd (1977) due to effortless remobilization of alkalis in pyroclastic material.

\section{VOLCANIC EVOLUTION OF THE COSIGÜINA VOLCANO}

\section{Pre-historic evolution of the Cosigïina peninsula}

The basement of the Cosigüina peninsula mostly consists of deeply weathered welded ignimbrites and lavas of the Coyol Formation. The basement is well exposed in the range of Loma El Ojochito (Figs. 2 and 3) and its occurrence continues to the 
mainland to the SE. A small block of the Coyol Formation also crops out at the foothill of the San Juan volcano (Fig. 3).

Two volcanic edifices representing Pleistocene volcanic activity cover the basement of the peninsula. These two eroded volcanoes occupy the major part of the peninsula, where the modern Cosigüina shield only represents the youngest stage of the volcanic system evolution.

San Juan volcano occupies the SE slopes of the Cosigüina Volcano, south of Potosí. Hradecký (1988) and van Wyk de Vries (1993) used the alternative name "Pleistocene volcano" for this apparatus. The stratovolcanic sequence is well shown in the northern side of the edifice, where non-welded, diagenetically indurated pyroclastic flow deposits contain abundant andesitic lithics. These deposits rest on the weathered lavas of the Coyol Formation in the southern part. Post-volcanic collapses deposited unsorted coarse debris flow accumulations, as is seen in a new road cut south of Potosí. The central part of the moderately eroded volcano consists of lavas with intercalations of proximal scoriae. San Juan volcano can be interpreted as an old shield volcano, probably of the same age as Pre-Cosigüina.

The term "Pre-Cosigüina" has been applied for lavas and associated pyroclastics that formed the original volcano, now preserved in the form of the relict of somma Cresta Montosa (Williams, 1952), better seen in the crater wall sections. Most of the area of the morphological somma is covered by dense vegetation and together with the older collapse structure of La Salvia, is blanketed by young tuffs. The structure can be identified by using aerial photographs. Pre-Cosigüina is considered as a relict of a probably Pleistocene shield volcano, which preceded the young cone.

La Salvia dyke lies inside layered deposits of pyroclastic flows at the Pacific coast of the peninsula. Although it reaches only some $1.5 \mathrm{~m}$ in thickness, this prominent dyke can be traced in distance of up to $3 \mathrm{~km}$. The dyke is probably of similar age as Pre-Cosigüina volcano and it was exposed by selective erosion. The apparent recent position of this dyke within the deposits of modern Cosigüina could be explained by filling of the relief around the exposed dyke by historical pyroclastic flows.

\section{Monogenetic forms of uncertain age}

Two monogenetic forms are located on the foothills of the Cosigüina Volcano. Maar Barranco (Fig. 3) is a small structure at the northeastern slope of the massif, built up by a phreatomagmatic eruption producing tephra rich in juvenile lithics - basaltic andesite in composition. Base-surge and fall-out deposits forming the tuff-ring are diagenetically consolidated and overhang the plain. The stratigraphic relations of this maar remain unclear. The degree of erosion could correspond to late Pre-Cosigüina stage.

Another volcanic feature rests on the southern slope of the volcano, probably associated with the final activity of Pre-Cosigüina and San Juan volcanoes. Los Chacos scoria cones (Fig. 3) were originated by small-scale Strombolian to Hawaiian eruption set on a NE trending rupture. These cones reveal the most primitive magma composition of all volcanic centers in the peninsula.

\section{Pyroclastic sequences of modern Cosigüina Volcano}

The later activity is more explosive in character, while lavas are more abundant in previous stages of the volcano evolution, and missing in last volcanic events. The subrecent and recent activity of the Cosigüina Volcano could be subdivided into five explosive phases:

Sequence I: pyroclastic flows PK, UH 1.

Sequence II: pyroclastic flows IN, scoria and pumice falls EP, pyroclastic surges PN.

Sequence III: pyroclastic surges DS, scoria falls SE.

Sequence IV: pyroclastic flows PC, UH 2 and BA.

Sequence V: pyroclastic flows, surges and ash fall, activity of 1835 . 
The explosive activity of these eruptive events was phreatic, phreatomagmatic and moderate plinian. The 1835 eruption seems to be relatively of small-scale in comparison with those preceding, judging from the thickness and distribution of the youngest tuffs. All defined sequences are separated one to other by erosional unconformities.

\section{Sequence I}

Prominent deposits of poorly welded pyroclastic flows can be traced along the northwest coast of the Cosigüina peninsula close to El Rosario. Thickness reaches up to $5 \mathrm{~m}$ without known bottom. These $\mathrm{UH}$ 1 deposits consist of basaltic lithics enclosed in an ash-matrix and often arranged in units tracing velocity gradients in a laminar flow (Fig. 6a). Greyishpink color is characteristic for these deposits and allows recognizing and correlating them. A typical feature is the abundance of carbonized tree-trunks (Fig. 6b) up to several meters long. Degassing pipes are abundant and concentrated above tree-trunks. Broken animal bones reported by Hradecký (1988) found at the bottom together with trees, can be taken as an evidence for a sudden volcanic blast, which destroyed forests and wildlife.

Radiometric data of charcoals from this deposit yielded approximately 500 B.P., suggesting eruption around the beginning of 16th century. Original $14 \mathrm{C}$ data from the deposits UH 1 (Sequence I) were calibrated by OxCal software (Ramsey, 2003; Table 1.).

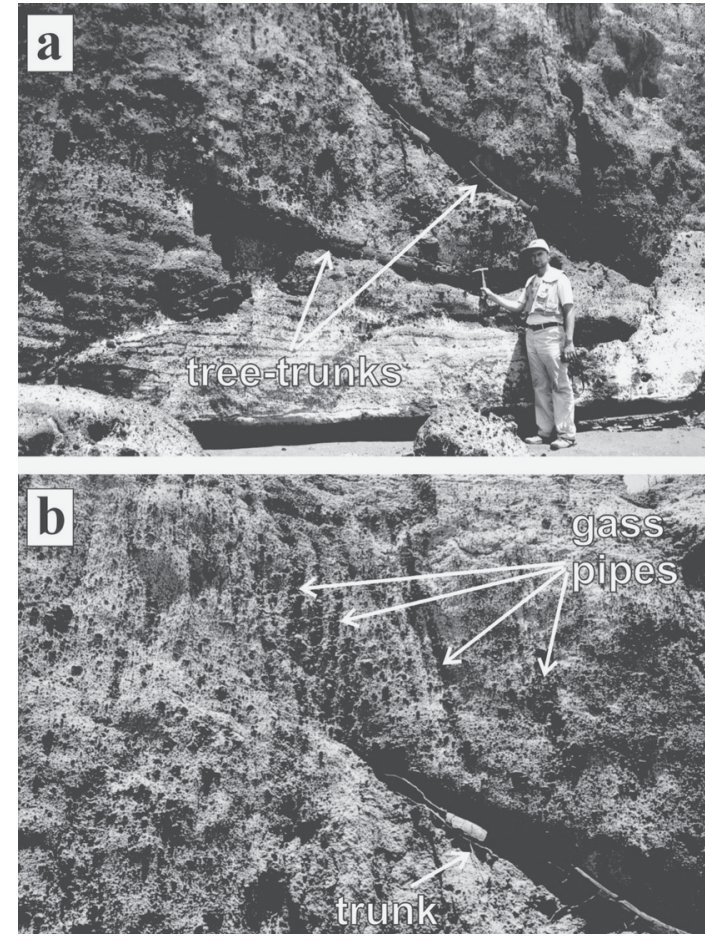

Fig. 6: a) Deposits of UH 1 pyroclastic flows of Sequence I. b) Carbonized tree-trunks up to $2 \mathrm{~m}$ long in deposits of UH 1 pyroclastic flows. Degassing pipes concentrate above trunks.

Thick deposits of PK pyroclastic flows crop out west of Ojo de Agua village (northwest of Punta Nata). Poorly welded and solidified deposits up to $12 \mathrm{~m}$ thick are overlain by DS surges. The matrix

Table 1

Calibration results of Sequence I radiocarbon data

\begin{tabular}{|c|c|c|c|c|}
\hline Non-calibrated data & $\begin{array}{c}\text { Probability } \\
64.1 \%\end{array}$ & $\begin{array}{c}1 \mathrm{~s} \\
4.1 \%\end{array}$ & $2 \mathrm{~s}$ & Calibrated ages \\
\hline \multirow[t]{2}{*}{$\begin{array}{l}498 \pm 127 \mathrm{BP} \\
\text { (PK) }\end{array}$} & 1300AD-1520AD & 1590AD-1620AD & 1260AD-1670AD & $1465 \pm 205 \mathrm{AD}$ \\
\hline & $65.0 \%$ & $3.2 \%$ & & \\
\hline \multirow[t]{2}{*}{$\begin{array}{c}505 \pm 127 \mathrm{BP} \\
\text { (UH 1) }\end{array}$} & $1290 \mathrm{AD}-1520 \mathrm{AD}$ & 1600AD-1620AD & 1260AD-1670AD & $1465 \pm 205 \mathrm{AD}$ \\
\hline & $65.1 \%$ & $3.1 \%$ & & \\
\hline $\begin{array}{c}506 \pm 127 \mathrm{BP} \\
(\mathrm{UH} 1)\end{array}$ & 1290AD-1520AD & 1600AD-1620AD & 1260AD-1670AD & $1465 \pm 205 \mathrm{AD}$ \\
\hline
\end{tabular}


corresponds to a basaltic andesite. Carbonized wood provided material for $14 \mathrm{C}$ data, which yielded close to UH 1 charcoals (Table 1), therefore this unit has been included, together with UH 1, to the Sequence I as the oldest exposed pyroclastic deposits of the modern volcano.

\section{Sequence II}

This sequence comprises mostly plinian deposits, which have been documented in the Tigüilitada, Punta El Papayal and La Salvia sections in coastal cliffs (northwest coast between Punta Nata and La Salvia). The block-and-ash pyroclastic flow and surge deposits (IN) are exposed in La Salvia, being $5 \mathrm{~m}$ thick (Fig. 7). The overlying phreatomagmatic unit PN consists of andesitic scoria and surge deposits in Tigüilitada section (between La Salvia and El Carmen) whereas in Apascalí, the profile is represented by air fall and mudflows.

The uppermost unit of Sequence II consists of light-grey pumice and repeated mingled magma-clast layers (EP, Fig. 8). Pumice corresponds to dacite as most silicic product of all Cosigüina analyzed rocks (Table 2).

\section{Sequence III}

Scoria falls on the SE can be best documented in the Tigüilitada section (between La Salvia and El Carmen, Fig. 8). Andesitic scoria reaches up to $3 \mathrm{~m}$ thick and was produced by moderate phreatomagmatic activity. The ages of Sequences II and III should be estimated on the basis of superposition as anytime between early 16th and early 17th century. Therefore, deposits of these two sequences could be assigned to the eruption in 1609 , which is commonly referenced as uncertain (Global Volcanism Program). The associated, $10 \mathrm{~m}$-thick surge and flow deposits DS (Figs. 9 and 10), of basaltic or andesitic composition, increase in thickness towards the NE. This well-preserved and distinctive unit can be traced in all documented sections - Tigüilitada, Apascalí and La Salvia.

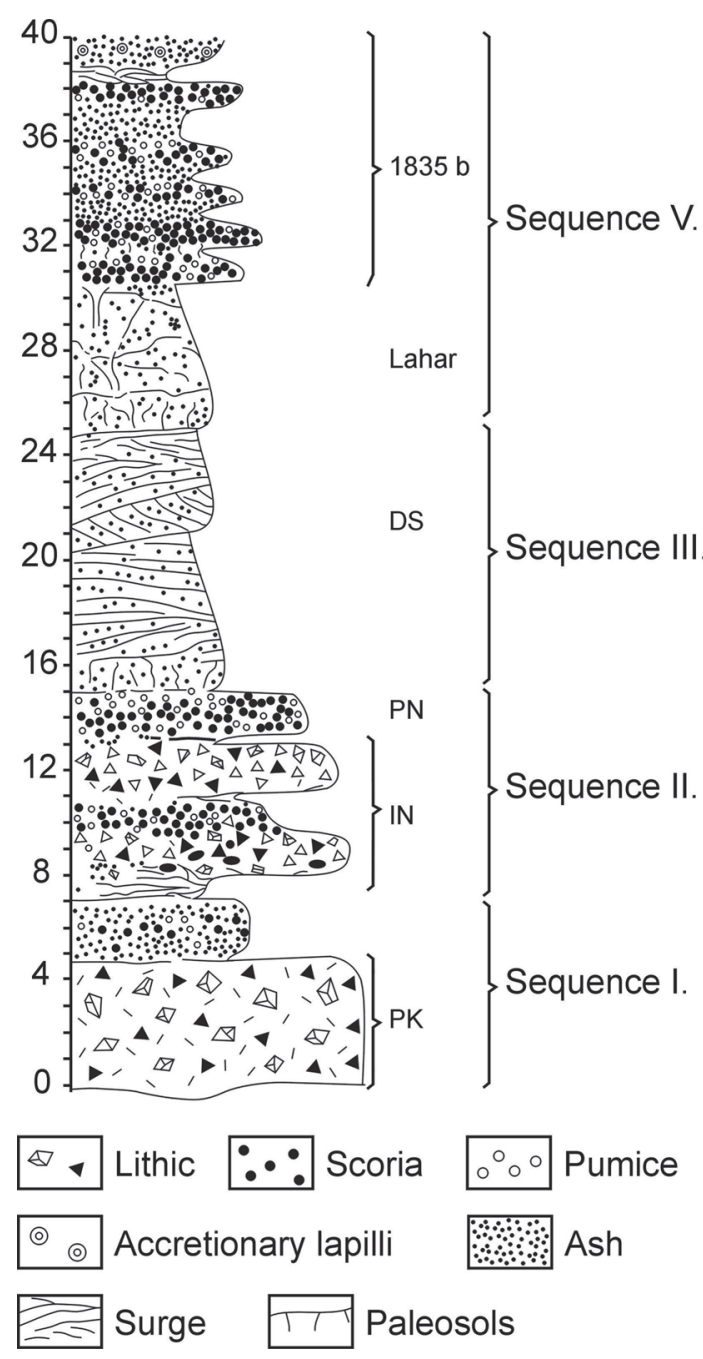

Fig. 7: Pyroclastic succession in the area of La Salvia, where sequence IV does not crop out. Abbreviations correspond to the names of pyroclastic units used in the text..

\section{Sequence IV}

There is an erosional unconformity between deposits of DS and Sequence IV (weathered surface). PC pyroclastic flows initiated the activity of this sequence. Deposits of PC are discontinuous and widespread covered UH2 pyroclastic flows (Fig. 11). The latter ones are represented by coarse-grained, non-welded, but well-cemented pyroclastic 


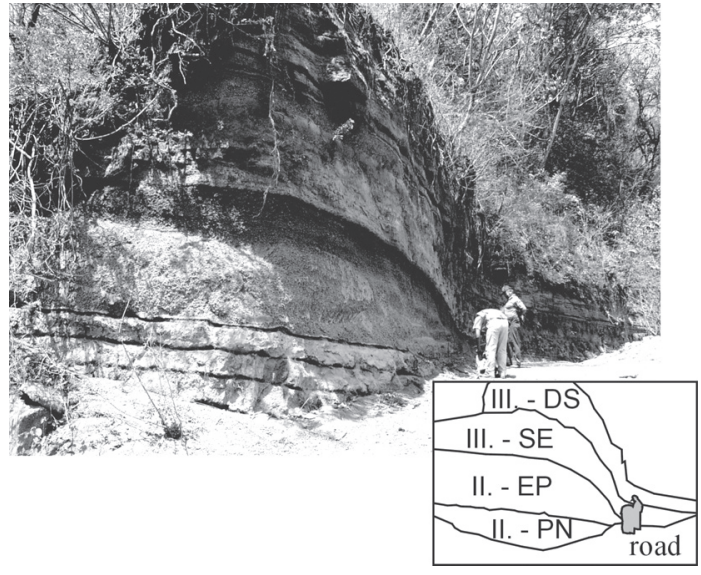

Fig. 8: Section at Tigüilitada. Silicic pumice of EP (in the middle) grades continuously to the darker scoria (magma zoning). Scoriae SE (Sequence III) overlie a paleosol of EP. Deposits of "dramatic surges" DS (Sequence III) are well exposed in the upper part of this section.

flow deposits occasionally containing fragments of carbonized wood. Calibration of radiocarbon data from the Sequence IV eruption were not possible. On the other hand, reviews on historic reports (Incer, 1983, 1988; Global Volcanism Program) present the possible eruption in 1709. This date well fits to our radiometric data (Table 1; Fig. 5).

The last unit BA forms parts of the coastal cliffs close to La Salvia. Some equivalents of the sequence were found also in the eastern side of the peninsula (San Marcos and Aguas Calientes, south of Potosí). All deposits are block-and-ash flows and scarce co-ignimbritic fallout tuffs.

\section{Sequence V (1835 AD)}

This sequence corresponds to the well-known eruption in 1835. A lot of good exposures have been documented, such as in Santa Elena, Apascalí, Tigüilitada, La Salvia, etc. The maximum thickness is about $8 \mathrm{~m}$. The lower part (1835a) is composed of scoria fall (probably a plinian blast), whereas scoriaceous pyroclastic flows of andesitic composition dominate the upper part (1835b).

The uppermost part of the sequence is represented by fine co-ignimbrite ashes of a typical yellowish color, that reach up to $2 \mathrm{~m}$ between
Punta Nata and Apascalí. On the other hand, irregular mudflows form the base of the deposit. Wide and short degassing pipes perpendicular to the surface of mudflow served as conduits for lukewarm water steam. Coarse-grained and poorly sorted lag-fall breccias surround the crater, showing good welding.

The activity of 1852 and 1859 , reported by Incer $(1983,1988)$ and the Global Volcanism

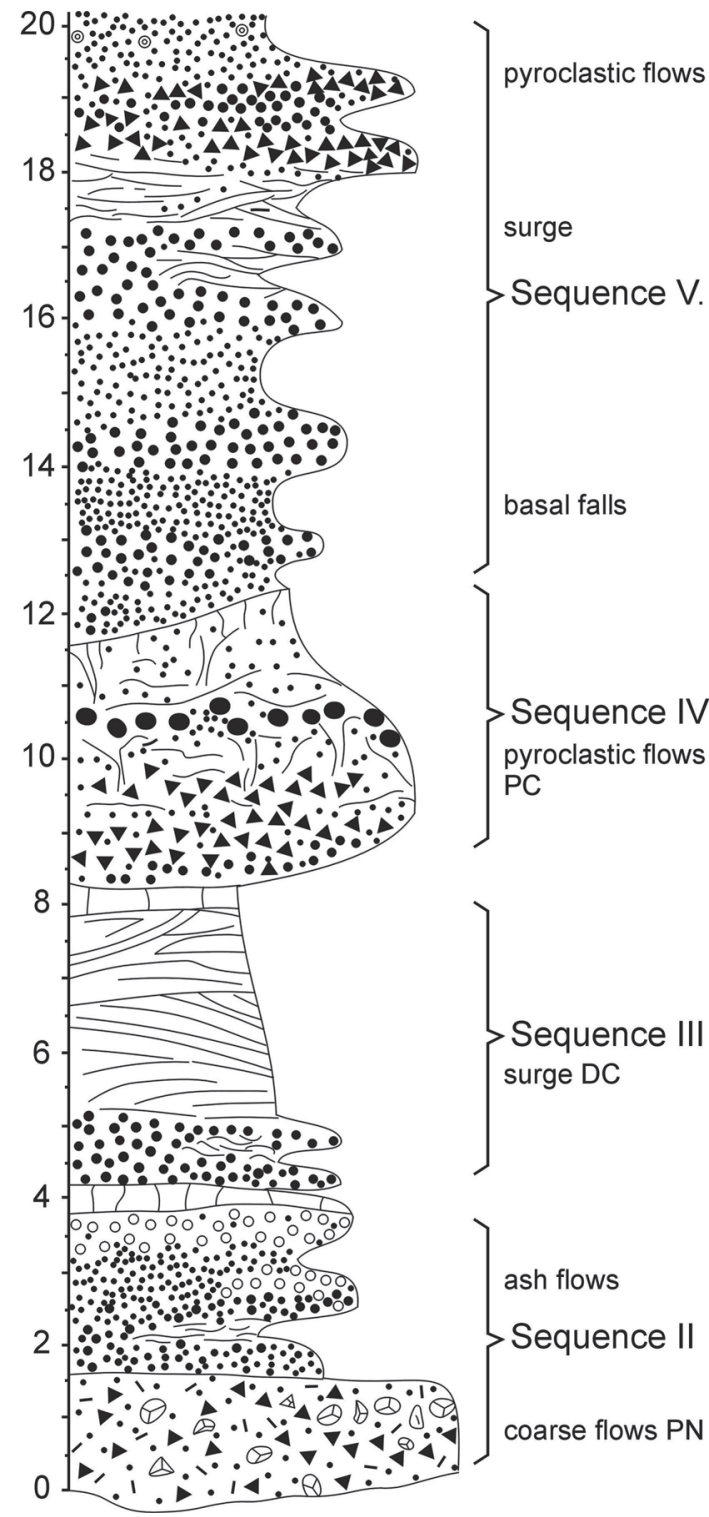

Fig. 9: Pyroclastic succession in the area of Apascalí. 


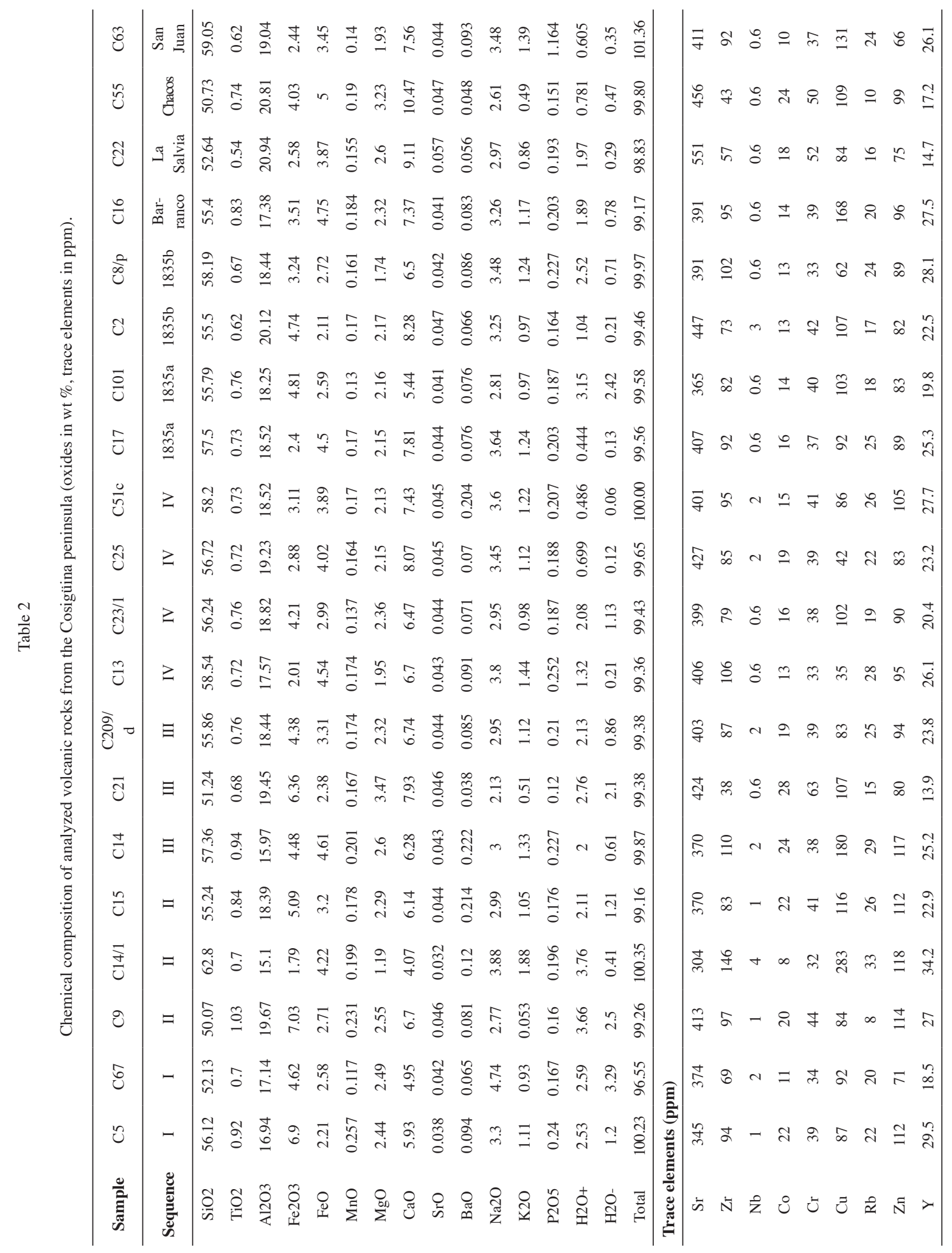


Program, most probably correspond to minor ash eruptions not preserved till nowadays. Bushfires could also explain these poorly described events.

Only few small fumaroles in the crater-lake and thermal water springs at the eastern feet of the edifice represent the recent activity.

\section{COMPOSITION OF ERUPTED MAGMAS}

The erupted magmas of the Cosigüina Volcano are relatively monotonous in composition. Most of the analyzed samples from the eruptive sequences have basaltic andesitic to andesitic composition. The studied samples were classified using the IUGS recommended TAS diagram (Le Bas et al., 1986; Fig. 12a). Since the pyroclastic material is prone to hydration and other postmagmatic alterations affecting predominantly the alkali contents, the classification was further confirmed in the diagram of Winchester \& Floyd (1977; Fig. 12b). No big differences in the resulting classification were revealed, denoting low alteration or low

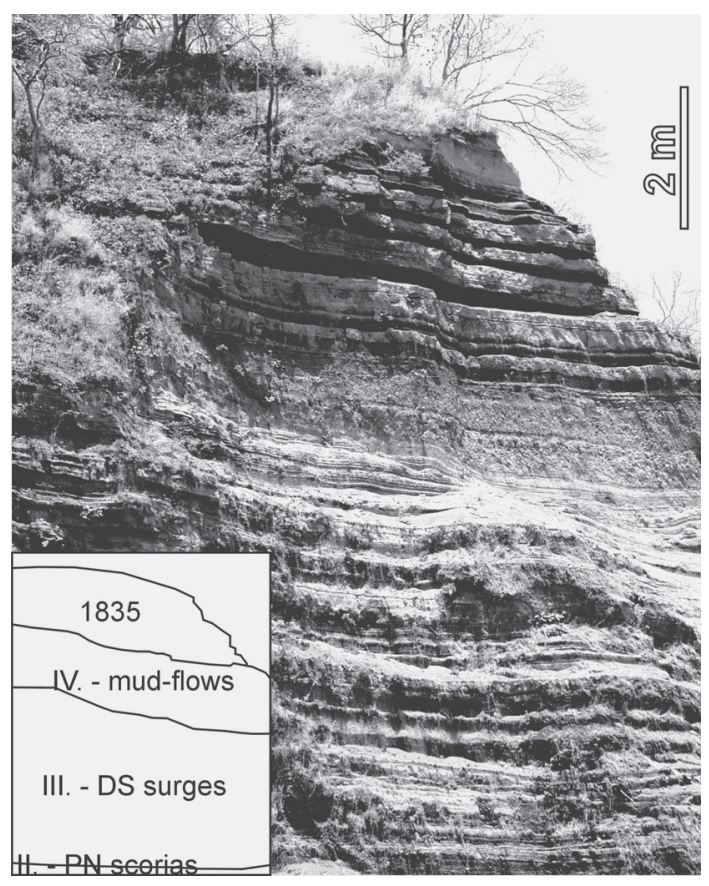

Fig. 10: Section east of La Salvia with complete succession of pyroclastic products of Sequence V (1835 AD) on top.

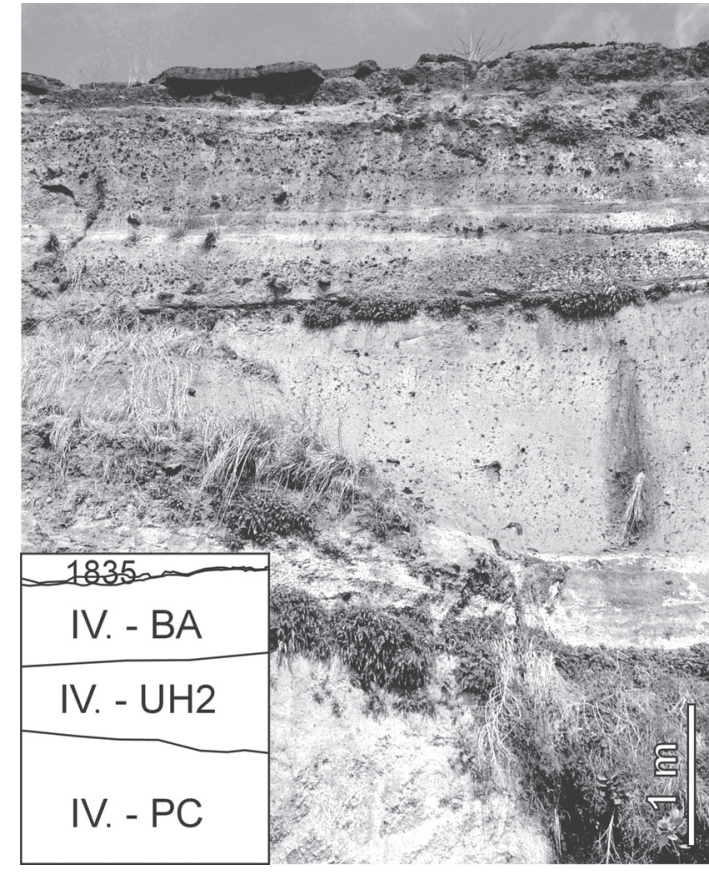

Fig. 11: Deposits of pyroclastic flows of Sequence IV on the NW coast of the peninsula. At the base, non-welded deposits of pyroclastic flows (PC), middle part consists of cemented pyroclastic flow deposits (UH2) and the sequence is crowned with block-and-ash BA deposits. Thin layer of 1835 eruption covers the deposits of Sequence IV.

depletion in mobile elements, including samples relatively rich in water. The only exception is the sample C67 slightly enriched in Na due to impregnation of porous pyroclastic material with seawater salts (outcrops are in the reach of tides).

Mid-K and calc-alkaline character of volcanic material produced from the Cosigüina Volcano corresponds to an active continental margin (Figs. $12 \mathrm{c}$ and 12d). The observed trends are in good agreement with data from other volcanoes of the Nicaraguan front (database of Carr et al., 2003), although in some cases the scatter of the present data is more pronounced than in the whole reference dataset. Although the data for some main oxides are scattered and the trends are not clear, a general decrease in compatible element contents such as $\mathrm{Mg}, \mathrm{Ca}, \mathrm{Fe}, \mathrm{Ti}, \mathrm{Al}$ as well as $\mathrm{Sr}$ and $\mathrm{Cr}$ may be observed, accompanied by the increase in incompatible element contents, such as $\mathrm{K}, \mathrm{Na}$, 


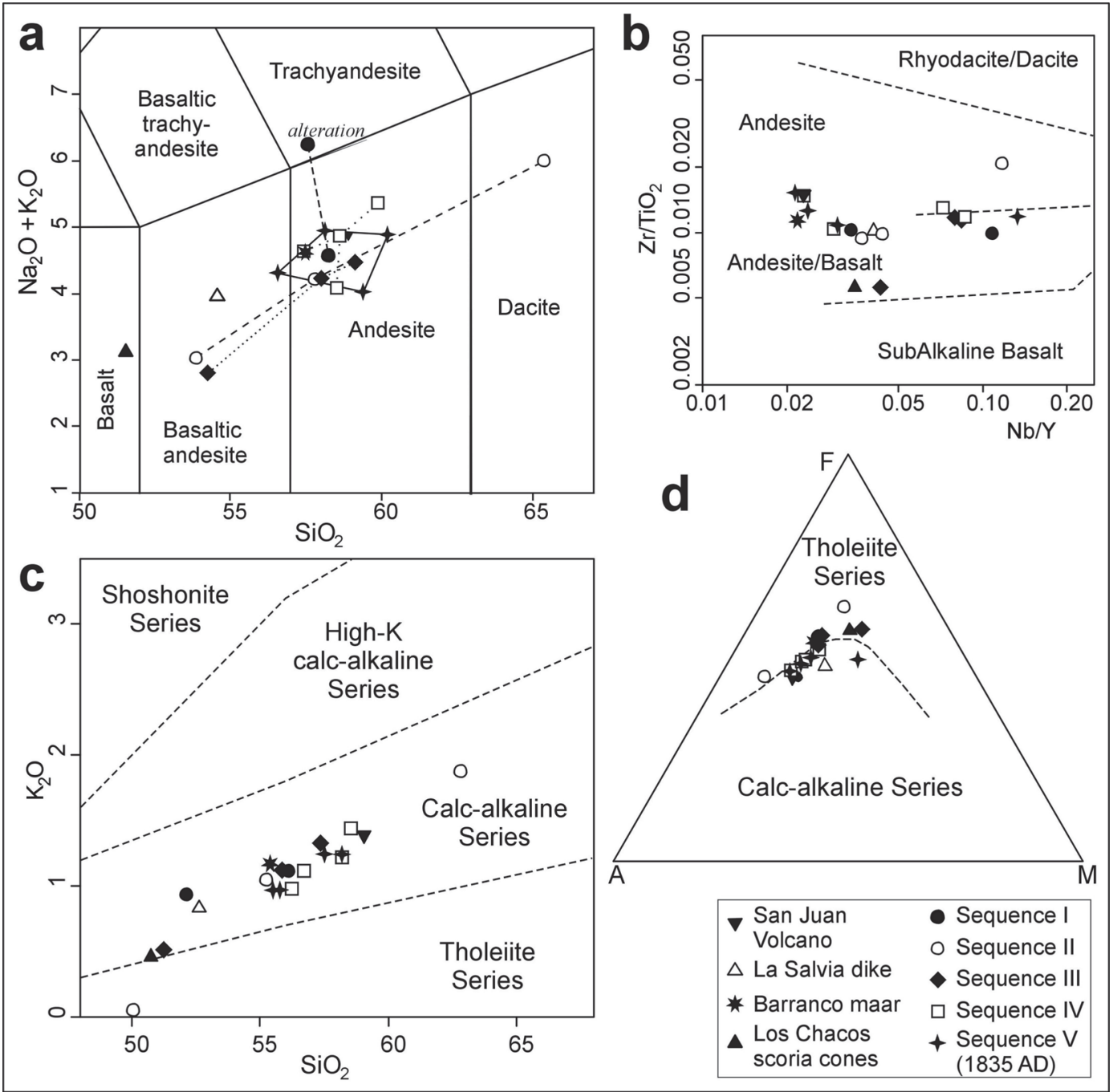

Fig. 12: a) TAS diagram (Le Bas et al., 1986) with rocks of the Cosigüina Peninsula; b) Nb/Y - Zr/TiO2 diagram (Winchester \& Floyd, 1977) based on immobile elements, more applicable to hydrated pyroclastic material; c) SiO2-K2O diagram (Peccerillo \& Taylor, 1976); d) AFM diagram (Irvine \& Baragar, 1971) of the Cosigüina volcanic rocks showing the calc-alkaline character overlapping the field of tholeiitic rocks.

$\mathrm{Rb}, \mathrm{Zr}$ and $\mathrm{Y}$. Such trends may be attributed to a common fractional crystallization of anorthitic plagioclase, iron rich olivine and/or clinopyroxene and Ti-magnetite. However, the detailed compositional trends within individual suites are far from linearity. Only the Sequence II shows a more significantly zoned reservoir together with an almost linear compositional trend for all main oxides. The span between the most primitive sample and the most evolved one, corresponds to ca $30 \%$ of fractional crystallization. This activity had finished with dacitic pumiceous fallout deposits of the EP unit (sample C14/1).

A singular sample demonstrates the andesitic composition of the Pleistocene San Juan shield volcano. As this study has not been focused on this extinct volcano, only one sample of this volcano has been analyzed. 
The variation in composition of the Pleistocene rocks is similar to the recent Cosigüina, ranging from basaltic andesites to andesites, except for the very primitive Cerros los Chacos scoria cones. The andesitic composition of the juvenile scoria of the El Barranco maar (sample C16 in the Table 2) is very similar to the composition of most of the pyroclastic material erupted from the Cosigüina Volcano. The dike of La Salvia is more basic (basaltic andesite), but also yields into the composition range of Cosigüina products.

Scoriae of the Cerros Los Chacos cinder cones are more mafic (basaltic). These should represent some more primitive magma, which probably never reached the main magma chamber. Their position on the SW-NE trending faults suggests a relation to the Guayape fault system, which probably enabled a rapid ascent of the melt.

\section{DISCUSSION AND CONCLUSIONS}

After a Pleistocene volcanic activity forming Pre-Cosigüina and San Juan shield volcanoes, a period of more explosive events took place. Five large eruptions are characteristic for the evolution of the Cosigüina volcano within the last 500 years. Phreatic, phreatomagmatic and moderate plinian eruptive styles characterized these last volcanic events of modern Cosigüina. Phreatic influence strongly increased the explosivity of andesitic (and scarcely dacitic) magma. The last eruption in 1835 was not the most violent one. Phreatic eruptions of high explosive energy took place when seawater infiltrated along fault zones (probably related to the Guayape fault system) to the magma reservoir. Such process could cause another violent phreatic or phreatomagmatic eruption in the future. Pyroclastic flows and fallout would endanger villages and the seaport of Potosí on the Cosiguiina peninsula.

\section{ACKNOWLEDGMENTS}

We gratefully acknowledge the Czech Ministry of Foreign Affairs and the Ministry of Environment of the Czech Republic for the financial support of the study within the framework of "Development assis- tance projects". Authors would like to give thanks to all field co-workers (in alphabetical order): Havlíček P., Hrubeš M., Mrázová Š., Opletal M., Šebesta J. and Ševčík J., as well as to laboratory analysts of the Czech Geological Survey and to Dr. Melková from Charles University (Prague) for 14C datings. The field campaign in Nicaragua was logistically supported by INETER, W. Strauch and E. Mayorga from the Department of Geophysics and Geology of this institution. Thanks to the anonymous reviewer.

\section{REFERENCES}

BURKART, B. \& SELF, S., 1985: Extension and rotation of crustal blocks in northern Central America and effect on the volcanic arc.- Geology, 13: 22-26.

CATASTRO e INVENTARIO de RECURSOS NATURALES, 1972: Geologic map. Sheet ND 16-14; Plate IV-3D; Chinandega, Nicaragua; Honduras.

FINCH,R.C.\& RITCHIE,A.W., 1991: The Guayape fault system, Honduras, Central America.- J. South Amer. Earth Sci. 4(1-2): 43-60.

\section{GLOBAL VOLCANISM PROGRAM -} SMITHSONIAN National Museum of Natural History: Cosigüina.- URL: http:// www.volcano.si.edu/world/volcano. cfm?vnum=1404-01=

HRADECKÝ, P., 1988: Los aspectos geológicos de la 1835 erupción del Volcán Cosigüina, Nicaragua. 38 págs.- Archive INETER, Managua [unpublished report].

HRADECKÝ, P., HAVLÍČEK, P., MAYORGA, E., OPLETAL, M., RAPPRICH, V., ŠEBESTA, J. \& ŠEVČÍK, J., 2001: Geological study: Research on natural hazards and vulnerability of geological environment in the region of the Cosiguiina volcano. (in Czech and Spanish). 36 págs.- Archives INETER Managua and CGS, Prague [unpublished report]. 
INCER, J., 1983: Crónicas sobre la gran erupción del Cosigüina.- La Prensa, 23th January 1983, Managua.

INCER, J., 1988: Central American volcanic events (1524-1924).- 52 págs. [unpublished manuscript].

IRVINE, T.N. \& BARAGAR, W.R.A., 1971: A guide to the chemical classification of the common volcanic rocks.- Can. J. Earth. Sci. 8: 523-548.

JANOUŠEK, V., FARROW, C. \& ERBAN, V., 2006: Interpretation of whole-rock geochemical data in igneous geochemistry: introducing Geochemical Data Toolkit (GCDkit).- J. Petrol. 47(6): 1255-1259.

Le BAS, M.J., Le MAITRE, R.W., STRECKEISEN, A. \& ZANETTIN, B., 1986: A chemical classification of volcanic rocks based on the total alkali-silica diagram.- J. Petrol. 27: 745-750.

MANN, P. - ed., 1995: Geologic and Tectonic Development of the Caribbean Plate Boundary in Southern Central America.349 págs. Geol. Soc. Am. Spec. Paper 295.

MARTÍNEZ, J. \& VIRAMONTE, L., 1982: Geologia y riesgos volcánicos de Nicaragua.- 67 págs. Archive INETER, Managua [unpublished report].

McBIRNEY, A. \& WILLIAMS, H., 1965: Volcanic history of Nicaragua.- Univ. California Press 55: 1-65.

PECCERILLO, R. \& TAYLOR, S.R., 1976: Geochemistry of Eocene calc-alkaline volcanic rocks from the Kastamonu area, Northern Turkey.- Contrib. Mineral. Petrol. 58: 63-81.
RAMSEY, C.B., 2003: OxCal Program v 3.9 - University of Oxford - Radiocarbon Accelerator Unit.- URL: http://www.rlaha. ox.ac.uk/oxcal/oxcal.htm

SCOTT, W., GARDNER, C., DEVOLI, G. \& ALVAREZ, A., 2006: The A.D. 1835 eruption of Volcán Cosigüina, Nicaragua: A guide for assessing local volcanic hazards.Geol. Soc. Am. Spec. Pap. 412: 167-188.

SELF, S., RAMPINO, R. \& CARR, M.J., 1989: A reappraisal of the 1835 eruption of Cosigüina and its atmospheric impact. Bull. Volcanol. 52: 57-65.

STUIVER, M., REIMER, P.J. \& BRAZIUNAS, T.F., 1998: High-precision radiocarbon age calibration for terrestrial and marine samples.- Radiocarbon 40(3): 1127-1151.

Van WYK De VRIES, B., 1993: Tectonics and magma evolution of Nicaraguan volcanic system.- 328 págs. Dept. of Earth Sciences, Open University, Milton Keynes [PhD. thesis].

WEYL, R., 1980: Geology of Central America. 371 págs. Gebrüder Borntraeger, Berlin.

WILLIAMS, H., 1952: The great eruption of Coseguina, Nicaragua in 1835. - Univ. Calif. Pub. Geol. Sci. 29: 21-45.

WILLIAMS, R.L. - ed., 1972: The geology of western Nicaragua. - 221 págs. Parsons Corp. Final Technical Report [unpublished report].

WINCHESTER, J.A. \& FLOYD, P.A., 1977: Geochemical discrimination of different magma series and their differentiation products using immobile elements. Chem. Geol. 20: 325-343. 